\title{
Optical Preparation and Coherent Control of Ultrafast Nonlinear Quantum Superpositions in Exciton Gases: A Case Study for Atomically Thin Semiconductors
}

\author{
Florian Katsch $\odot^{*}$ and Andreas Knorr \\ Institut für Theoretische Physik, Nichtlineare Optik und Quantenelektronik, \\ Technische Universität Berlin, 10623 Berlin, Germany
}

(Received 28 May 2020; revised manuscript received 29 August 2020; accepted 12 October 2020; published 24 November 2020)

\begin{abstract}
We report on the optical preparation of coherent superpositions of exciton and biexciton states manifested in temporal nonlinear oscillations in interacting exciton gases. The effect is illustrated for atomically thin semiconductors, where the reflected light reveals these interactions in a unique way. The occurring nonlinear coherent oscillations are counteracted by incoherent excitation-induced dephasing, a phenomenon which originates from a new type of quantum interference between excitons and the twoexciton scattering continuum. To improve the experimental accessibility, we discuss different methods to control the oscillation modulation depth by modifying the mutual interplay of the exciton-biexciton superposition and excitation-induced dephasing. We find that the coherent optical response can be manipulated by the polarization degree of the exciting light field, the laser detuning, external magnetic fields, and quantum coherent feedback. The extraordinary temporal behavior and its control distinguishes the nonlinear coherent oscillations from atomic Rabi oscillations and enables their engineering based on our proposed control schemes.
\end{abstract}

DOI: $10.1103 /$ PhysRevX.10.041039

Subject Areas: Optoelectronics, Semiconductor Physics

\section{INTRODUCTION}

The coherent manipulation of the superposition of quantum states plays a central role for many novel, especially quantum technological, applications [1]. A typical example constitutes an atomic two-level system where the electrical field controls the coherent superposition of upper and lower state populations, observed as Rabi oscillations [2]. Rabi oscillations constitute a fundamental effect of coherent light-matter interaction induced by the Pauli blocking nonlinearity. Rabi oscillations describe a sinusoidal time evolution of the population difference in a two-level system controlled by the area $\Theta=$ $\int_{-\infty}^{\infty} d t \Omega(t) \geq 2 \pi$ of the optical pulse [3]. The Rabi frequency $\Omega(t)=d E_{0}(t) / \hbar$ is defined by the transition dipole element $d$ of the two-level system and the external optical field $E_{0}(t)$. The simplest description for two-level systems results in harmonic oscillations of the upper-level occupation $n(t)=\sin ^{2}[\Omega t / 2]$ for resonant excitation with a square pulse $E_{0}(t)=$ const [3]. The direct control of these oscillations by the pulse area is established for two- and multilevel systems, such as atomic systems [3,4] and

\footnotetext{
*fkatsch@itp.tu-berlin.de
}

Published by the American Physical Society under the terms of the Creative Commons Attribution 4.0 International license. Further distribution of this work must maintain attribution to the author(s) and the published article's title, journal citation, and DOI. artificial atoms like semiconductor quantum dots [5-14] constituting qubit control by electronic Pauli blocking in quantum information science [15-18].

The search for coherent nonlinearities beyond strongly localized electronic excitations and beyond Pauli blocking directly leads to excitons as single, quasiparticle excitations, which are built up from Coulomb bound electronhole pairs, for instance, provided by atomically thin semiconductors, mesoscopic semiconductor quantum wells, and bulk semiconductors [19]. The steering of these excitonic quantum states requires the control of intriguing many-body effects on ultrashort timescales due to nonlinear exciton-exciton interactions. The latter include, but are typically not dominated by, Pauli blocking. Previously, the coherent manipulation of pure excitonic quantum states in conventional semiconductor quantum wells was obscured by electron-hole continuum transitions due to small exciton-binding energies [20-30]. However, recent progress in the fabrication of atomically thin semiconductors with reduced environmental screening [31] can be used to optically prepare almost pure exciton gases. A typical example constitutes a monolayer of the transition metal dichalcogenide $\mathrm{MoS}_{2}[32,33]$ with exceptionally large exciton-binding energies separated hundreds of $\mathrm{meV}$ from the band gap [34-37]. Once optically excited close to the exciton resonance, the large energy separation efficiently suppresses electron-hole continuum transitions above the band gap. This suppression results in almost pure excitonic nonlinearities [38-43] which—already to lowest order-are influenced by 
strong many-exciton interactions. In particular, quantum superpositions of excitons lead to bound two-exciton states, referred to as biexcitons, and corresponding two-exciton scattering continua. Whereas biexcitons form another energetically localized excitation beyond the exciton, twoexciton scattering continua induce a prototype of quantum interference due to many dense states. The latter produces a pronounced power-dependent dephasing $[44,45]$ referred to as excitation-induced dephasing. This result connects the two central research areas of coherence and decoherence in excitonic, quasibosonic quantum gases.

In this paper, we explore the occurrence and coherent superposition of optically induced exciton density oscillations resulting from excitonic nonlinearities. The excitonic nonlinearities are provided by atomically thin semiconductors characterized by interactions of excitons, biexcitons, and the two-exciton scattering continua. Coulomb correlations induce oscillations which resemble a nonlinear excitation transfer between excitons and biexcitons counteracted by and interplaying with excitationinduced dephasing. Since the oscillations originate from Coulomb correlation effects, they contrast Rabi oscillations induced by Pauli blocking nonlinearities. In particular, the different nature and properties of the observed oscillations in atomically thin semiconductors identify fundamental differences to excitons in semiconductor quantum dots $[6-8,15]$ and conventional semiconductor quantum wells $[23,25,26]$. The distinguished character of Coulomb-correlation-induced oscillations is also demonstrated by new control parameters which can be used to steer excitonic nonlinearities due to a detuning of the laser pulse energy, magnetic fields, and quantum coherent feedback.

This paper is organized as follows: We start in Sec. II by evaluating the excitonic Bloch equations [46,47], which incorporate exciton-exciton interactions beyond the meanfield approximation by explicitly including the nonlinear coupling of excitons to biexcitons and their two-exciton scattering continua [48-50]. Our energy- and time-resolved simulations in Sec. III for a monolayer of $\mathrm{MoS}_{2}$ as a prototypical semiconductor with tightly bound excitons reveal the suppression of Rabi-like mean-field-based oscillations due to excitation-induced dephasing (EID). We show that nonlinear coherent exciton oscillations beyond the mean-field limit are induced by exciton-exciton interactions for linearly polarized excitation of a monolayer of $\mathrm{MoS}_{2}$, stimulating an excitation exchange between excitons and biexcitons. However, these nonlinear coherent oscillations are still counteracted by incoherent EID originating from the two-exciton scattering continuum. Therefore, we subsequently explore the suppression of EID and the manipulation of the biexciton resonance in Sec. IV: First, in Sec. IV A, we investigate the influence of a laser detuning with respect to the exciton resonance energy. Depending on the chosen detuning, the ratio of excited biexcitons to two-exciton scattering continua can be manipulated, which allows one to enhance nonlinear coherent oscillations and reduce EID. Second, in Sec. IV B, we demonstrate that the excitation balance between biexcitons and the two-exciton scattering continuum can be manipulated by magnetic fields. Since the two-exciton scattering continuum induces EID, nonlinear coherent oscillations can be amplified or suppressed by a magnetic field. Third, in Sec. IV C, we employ quantum coherent feedback to steer the dynamics of exciton states in the presence of an external mirror reflection geometry. Such half-sided cavities constitute a model system for quantum optical feedback. Here, the spatial distance between the atomically thin semiconductor and the mirror introduces a time and phase delay which enables the manipulation of the biexciton energy and oscillator strength. Finally, we conclude by summarizing and presenting an outlook in Sec. V.

\section{OPTICALLY INDUCED NONLINEAR EXCITON-EXCITON INTERACTIONS}

To study purely excitonic nonlinearities, for instance, in atomically thin semiconductors with tightly bound excitons, we focus on the nonlinear optical response described by a purely excitonic theory [46,47]. This limit is reached in the weakly nonlinear, well-controlled excitation limit [51]. The geometry that we investigate is illustrated in Fig. 1(a). We start from the incoming light field with $\sigma_{ \pm}$circular polarization $\boldsymbol{E}_{ \pm}(t)$ or linear polarization $\boldsymbol{E}_{x}(t)$ describing plane waves propagating perpendicular $r_{\perp}$ to a semiconductor sample with the wave vector $q_{p}$ :

$$
\begin{aligned}
& \boldsymbol{E}_{ \pm}(t)=\frac{1}{2} E_{0, \pm}(t) e^{i q_{p} r_{\perp}} e^{-i \omega_{p} t} \mathbf{e}_{\sigma_{ \pm}}+\text {c.c. } \\
& \boldsymbol{E}_{x}(t)=\frac{1}{2} E_{0, x}(t) e^{i q_{p} r_{\perp}} e^{-i \omega_{p} t} \mathbf{e}_{x}+\text { c.c. }
\end{aligned}
$$

Here, $E_{0, \pm}(t)$ and $E_{0, x}(t)$ are the field envelopes, $\omega_{p}$ is the laser frequency, $\mathbf{e}_{\sigma_{ \pm}}=\left(\mathbf{e}_{x} \pm \mathbf{i} \mathbf{e}_{y}\right) / \sqrt{2}$ denote the normalized $\sigma_{ \pm}$circular polarization vectors, and $\mathbf{e}_{x / y}$ are the linear polarization vectors perpendicular to the propagation direction.

The incoming light field $E_{0, \pm}(t)$ induces a transmitted $E_{t, \pm}(t)$ and reflected $E_{r, \pm}(t)$ field at the monolayer position as illustrated in Fig. 1(a) [52,53]:

$$
\begin{aligned}
& E_{t, \pm}(t)=E_{0, \pm}(t)+i \alpha P_{ \pm}^{*}(t), \\
& E_{r, \pm}(t)=E_{t, \pm}(t)-E_{0, \pm}(t)=i \alpha P_{ \pm}^{*}(t) .
\end{aligned}
$$

$E_{t, \pm}(t)$ and $E_{r, \pm}(t)$ are obtained by solving Maxwell's wave equation in the thin sample limit, i.e., sample thickness $\ll \lambda_{p}=2 \pi c_{0} /\left(n_{r} \omega_{p}\right)$, where $\lambda_{p}$ is the wavelength of the incident light field depending on the vacuum speed of light $c_{0}$, the refractive index of the dielectric environment 
(a)

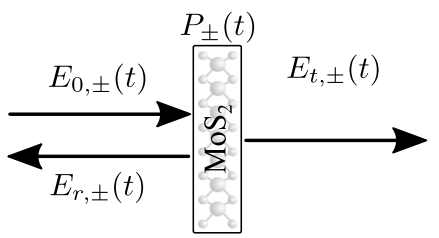

(b)

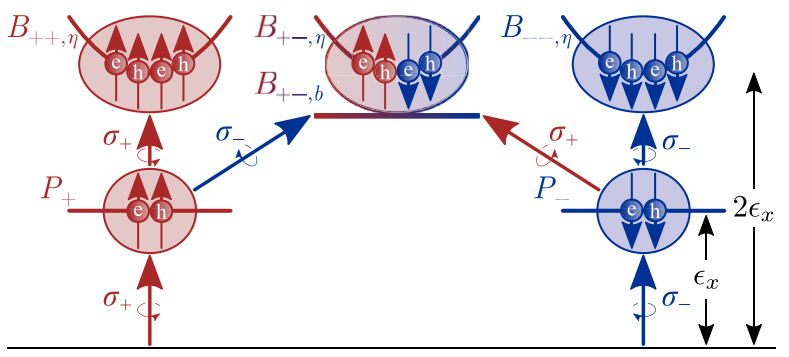

FIG. 1. (a) Illustration of the incoming light field $E_{0, \pm}(t)$ driving the exciton transitions $P_{ \pm}(t) . E_{0, \pm}(t)$ and $P_{ \pm}(t)$ determine the transmitted $E_{t, \pm}(t)$ and reflected $E_{r, \pm}(t)$ light fields. (b) Illustrated are excitons $P_{ \pm}$(bound electron-hole pairs with energy $\epsilon_{x}$ ), biexcitons $B_{+-, b}$ (two-electron and two-hole correlations with energy $<2 \epsilon_{x}$ ), and two-exciton scattering continua $B_{+-, \eta}$ and $B_{ \pm \pm, \eta}$ (two-electron and two-hole correlations with energy $\geq 2 \epsilon_{x}$ ). The optical selection rules for $\sigma_{+}$and $\sigma_{-}$circularly polarized light are indicated.

$n_{r}$, and the laser frequency $\omega_{p}$. The transmitted light $E_{t, \pm}(t)$ given in Eq. (2) couples to the polarization density $d^{*} P_{ \pm}(t)$ of the exciton gas and constitutes the light field at the position of the sample. The coefficient $\alpha=\omega_{p} d /\left(2 \varepsilon_{0} c_{0} n_{r}\right)$ involves the excitonic transition dipole element $d$ (see definition below) and the vacuum permittivity $\varepsilon_{0}$.

For resonant excitation of the energetically lowest $1 \mathrm{~s}$ exciton resonance, the response is dominated by the $1 \mathrm{~s}$ exciton transitions $P_{ \pm}$. Here, the index $+(-)$denotes the high-symmetry point $K\left(K^{\prime}\right)$ and corresponding spins $\uparrow(\downarrow)$ of the electrons and holes forming the exciton after ground state to single electron-hole pair excitation [47]. The exciton transitions $P_{+}$and $P_{-}$are illustrated as transition amplitudes to bound electron-hole pairs on the lower straight lines in Fig. 1(b). The excitonic response of the coherent dipole transitions $P_{ \pm}$is derived by employing a dynamics controlled truncation for weakly interacting excitons [51] to atomically thin semiconductors [50,54]:

$$
\begin{aligned}
{\left[\hbar \partial_{t}\right.} & \left.+\gamma_{0}+i\left(\hbar \omega_{p}-\epsilon_{x, \pm}\right)\right] P_{ \pm} \\
= & -\frac{i}{2} d E_{t, \pm}^{*}(t)+i \hat{d} E_{t, \pm}^{*}(t)\left|P_{ \pm}\right|^{2}+i \hat{V} P_{ \pm}\left|P_{ \pm}\right|^{2} \\
& +i \sum_{\eta}\left(W_{ \pm \pm, \eta} B_{ \pm \pm, \eta} P_{ \pm}^{*}+W_{+-, \eta} B_{+-, \eta} P_{\mp}^{*}\right) .
\end{aligned}
$$

Equation (4) describes excitonic oscillators $P_{ \pm}$with energy $\hbar \omega_{p}-\epsilon_{x, \pm}$ in the rotating frame of the laser frequency $\omega_{p}$. The exciton energies $\epsilon_{x, \pm}$ and wave functions $\varphi_{ \pm, q}$ are obtained by solving the Wannier equation for a monolayer of $\mathrm{MoS}_{2}$ [19]. This equation defines the excitonic transition dipole element $d=d^{v, c} \sum_{q} \varphi_{ \pm, q}$ with respect to the electronic dipole element $d^{v, c}$. Because of the optical selection rules in monolayers of $\mathrm{MoS}_{2}, P_{ \pm}$is coherently driven by the $\sigma_{ \pm}$circularly polarized light field $E_{t, \pm}^{*}(t)$ [55] illustrated as red and blue arrows in Fig. 1(b). The dipole oscillations are damped by a microscopically calculated phonon-mediated pure dephasing $\gamma_{0}$ [56-58]. A self-consistent solution of the Maxwell's and excitonic Bloch equations (2) and (4) [52,53] results in an additional radiative dephasing $\gamma_{r}=\alpha^{*} d$.

The second to fourth terms on the right-hand side of Eq. (4) constitute nonlinear contributions to the exciton dynamics, namely, Pauli blocking in the low-density limit $\sim E_{t, \pm}^{*}(t)\left|P_{ \pm}\right|^{2}$ [59], exciton-exciton interactions on a meanfield (Hartree-Fock) level $\sim P_{ \pm}\left|P_{ \pm}\right|^{2}$ (mean-field Coulomb coefficient $\hat{V}$ ), and the coupling of excitons $P_{ \pm}$to twoelectron and two-hole Coulomb correlations $B_{ \pm \pm, \eta}$ and $B_{+-, \eta}$ resulting in source terms $\sim B_{ \pm \pm, \eta} P_{ \pm}^{*}$ and $B_{+-, \eta} P_{\mp}^{*}$ (higher-order Coulomb correlation coefficients $W_{ \pm \pm, \eta}$ and $\left.W_{+-, \eta}\right)$, respectively. Without spin and polarization mixing at the $K$ and $K^{\prime}$ points, exciton-exciton interaction on a mean-field (Hartree-Fock) level $\sim P_{ \pm}\left|P_{ \pm}\right|^{2}$ mediates only intravalley scattering among exciton transitions $P_{+}$or $P_{-}$. Intervalley interactions, i.e., cross interactions, between $P_{+}$ and $P_{-}$on a mean-field level would require a Coulomb potential including electronic intervalley scattering cross sections which is typically negligible [54]. Therefore, the dominating contribution to intervalley interactions are due to terms $\sim B_{+-, \eta} P_{\mp}^{*}$ associated with the two-electron and two-hole Coulomb correlations $B_{+-, \eta}$.

The properties of $B_{ \pm \pm, \eta}$ and $B_{+-, \eta}$ are determined by the two-electron and two-hole Schrödinger equation [49,50]. Its solution provides bound states $\eta=b$ with energy $\epsilon_{x x,+-, \eta=b}<\epsilon_{x,+}+\epsilon_{x,-}$ (biexcitons) and unbound continuous states $\eta \neq b$ with energies $\epsilon_{x x, \pm, \pm, \eta \neq b} \geq 2 \epsilon_{x, \pm}$ and $\epsilon_{x x,+-, \eta \neq b} \geq \epsilon_{x,+}+\epsilon_{x,-}$ (two-exciton scattering continua). The system of Heisenberg equations of motion is closed by the equations for the Coulomb correlations $B_{\lambda_{1} \lambda_{2}, \eta}$ $\left(\lambda_{1}, \lambda_{2}= \pm\right)[51]$ described by phonon-damped $\left(2 \gamma_{0}\right)$ oscillators with energies $\epsilon_{x x, \lambda_{1} \lambda_{2}, \eta}$ which are purely Coulomb driven $\left(\hat{W}_{\lambda_{1} \lambda_{2}, \eta}\right)$ by two excitons $P_{\lambda_{1}} P_{\lambda_{2}}$ [54]:

$$
\begin{aligned}
& {\left[\hbar \partial_{t}+2 \gamma_{0}+i\left(2 \hbar \omega_{p}-\epsilon_{x x, \lambda_{1} \lambda_{2}, \eta}\right)\right] B_{\lambda_{1} \lambda_{2}, \eta}} \\
& \quad=i \hat{W}_{\lambda_{1} \lambda_{2}, \eta} P_{\lambda_{1}} P_{\lambda_{2}} .
\end{aligned}
$$

Two excitons with the same polarization $\sim P_{+} P_{+}$and $\sim P_{-} P_{-}$drive only the two-exciton scattering continua $B_{++, \eta \neq b}$ and $B_{--, \eta \neq b}$, respectively. These continua are illustrated as parabolas in Fig. 1(b). In contrast, two excitons with opposite polarization $\sim P_{+} P_{-}$simultaneously drive biexcitons $B_{+-, \eta=b}$ as two-exciton bound states and the two-exciton scattering continuum $B_{+-, \eta \neq b}$. Biexcitons $B_{+-, \eta=b}$ are drawn as a straight line and the two-exciton 
scattering continuum $B_{+-, \eta \neq b}$ as a parabola in the middle of Fig. 1(b). Since the repulsive interaction $P_{+} P_{+}$or $P_{-} P_{-}$ precludes biexcitons for excitation with $\sigma_{+}$or $\sigma_{-}$light solely [60-62], no straight lines are drawn below the parabolas on the left- and right-hand sides in Fig. 1(b).

The set of nonlinear equations of motion (4) and (5) already successfully explained optical wave mixing experiments performed on semiconductor quantum wells [63-66] and heterostructures of larger thickness $[67,68]$, as well as semiconductor microcavities [69-71], surfaces [72], and nanorings $[73,74]$. In particular, a nontrivial, nonperturbative treatment is valid in arbitrarily high order in the field $[75,76]$, provided that the exciton occupation is small enough to stay in the validity range [77]. The validity to use these equations to study nonlinear interactions for increasing field strength was already shown for excitationinduced dephasing [45].

Note that a residual electron or hole doping in atomically thin semiconductors due to external gate voltages [78], surface functionalization [79], and substrates [80], as well as impurities or vacancies [81], can induce trion resonances [82-88] occurring besides charge-neutral exciton resonances. The influence of typically energetically lower trions can be neglected as long as they are not resonantly excited and the optical pulses are short compared to the long trion formation times on the picosecond timescale [89-91]. Moreover, applying an external gate voltage allows one to suppress charge-tunable states such as trion resonances [92] and accesses charge-neutral atomically thin semiconductors, representing an excellent platform to study exciton-exciton interactions dominated by charge-neutral excitons and biexcitons [93-98].

\section{COHERENT OSCILLATIONS IN EXCITON GASES}

We self-consistently solve the Maxwell's and excitonic Bloch equations [Eqs. (2)-(5)] for a monolayer of $\mathrm{MoS}_{2}$ surrounded by hexagonal boron nitride at a temperature of $5 \mathrm{~K}$ as a prototypical atomically thin semiconductor with tightly bound excitons [99]. All used material parameters are summarized in the Appendix A.

\section{A. Spectral characterization}

To characterize the sample, the linear transmission spectrum $\sum_{ \pm}\left|E_{t, \pm}(\omega)\right|^{2} / \sum_{ \pm}\left|E_{0, \pm}(\omega)\right|^{2}$ is depicted as a black curve in Fig. 2(a). The exciton resonance exhibits a Lorentzian line shape including radiative $\left(2 \gamma_{r}=1.5 \mathrm{meV}\right)$ and phononinduced linewidth contributions $\left(2 \gamma_{0}=0.4 \mathrm{meV}\right.$ at $5 \mathrm{~K} \mathrm{[45]).}$

Note that we do not include an additional inhomogeneous broadening, and, thus, our results apply to high-quality samples: In particular, recent advances in fabricating highquality monolayers of $\mathrm{MoS}_{2}$ demonstrate the possibility to produce samples with low inhomogeneous broadening $[42,99]$. In contrast, we expect that the nonlinear coherent

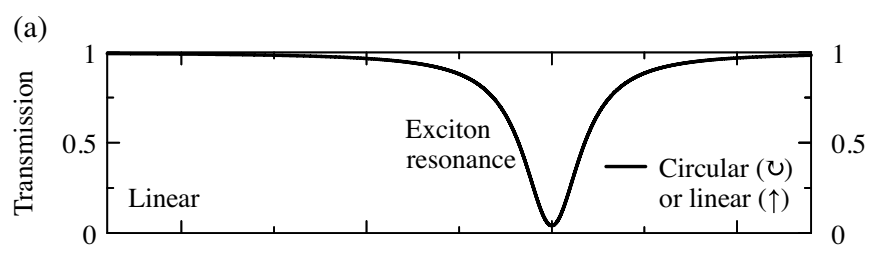

(b)

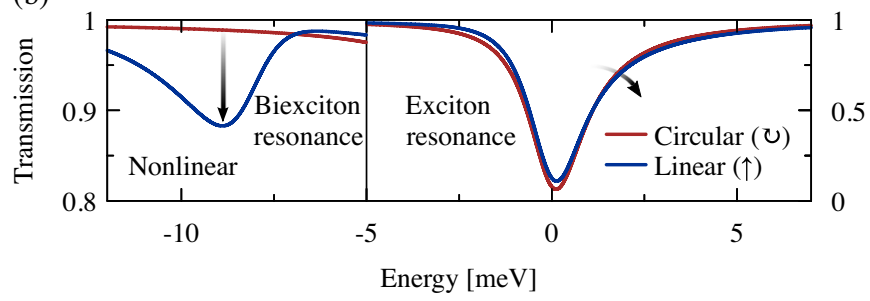

FIG. 2. (a) Linear (pulse area $\Theta \rightarrow 0$ ) and (b) nonlinear (pulse area $\Theta=0.1 \pi)$ transmission spectra near the exciton resonance $P_{ \pm}$for circularly (ひ) and linearly ( $\uparrow$ ) polarized excitation with 40 fs pulses (intensity FWHM) for a monolayer of $\mathrm{MoS}_{2}$ at $5 \mathrm{~K}$. The laser pump energy $\hbar \omega_{p}=\epsilon_{x, \pm}$ is resonant to the exciton resonance energy $\epsilon_{x, \pm}$.

oscillations cannot be observed for samples with large inhomogeneous broadening exceeding the biexciton-binding energy.

The linear response for negligible pulse areas $\Theta=\int_{-\infty}^{\infty} d t d E_{0, \pm}(t) / \hbar \rightarrow 0$, plotted as a black curve in Fig. 2(a), is equivalent for circularly and linearly polarized light. The incoming light fields are chosen to ensure the same intensity with respect to the $K$ point. In contrast, the nonlinear response for significant pulse areas $\Theta=0.1 \pi$ as plotted in Fig. 2(b) requires one to distinguish between (i) circularly and (ii) linearly polarized excitation.

(i) The red curve in Fig. 2(b) represents the transmission for $\sigma_{+}$circularly polarized excitation (ひ), exciting $P_{+}$solely. Compared to the linear transmission in Fig. 2(a), the exciton resonance shows a bleaching, a small blueshift, and the formation of a two-exciton scattering-continuum-induced sideband on the high-energy side of the exciton resonance. The sideband is coherently controlled by quantum interference between excitons $P_{+}$and the twoexciton scattering continuum $B_{++, \eta}$ inducing excitation-induced dephasing.

(ii) Linearly polarized light $(\uparrow)$ simultaneously excites $P_{+}$and $P_{-}$. Their attractive interaction drives the biexciton $B_{+-, b}$ along with the two-exciton scattering continua $B_{+-, \eta \neq b}, B_{++, \eta \neq b}$, and $B_{--, \eta \neq b}$; cf. Eq. (5). Therefore, biexciton resonances $B_{+-, b} P_{\mp}^{*}$ appear in the nonlinear transmission spectrum plotted as a blue curve in Fig. 2(b). [The transmission around the biexciton resonance in Fig. 2(b) is 5-times increased.] Note that we distinguish between biexcitons and biexciton resonances: Biexcitons $B_{+-, b}$ with energies $\epsilon_{x x,+-, b}$ are governed by Eq. (5). 
The associated biexciton resonances $B_{+-, b} P_{\mp}^{*}$ with energies $\epsilon_{x x,+-, b}-\epsilon_{\mp, x}$ couple to the exciton transitions $P_{ \pm}$by appearing on the right-hand side of Eq. (4). Therefore, biexciton resonances $B_{+-, b} P_{\mp}^{*}$ are visible as resonances in the blue curve in Fig. 2(b) below the exciton energies: $\epsilon_{x x,+-, b}-\epsilon_{\mp, x}<\epsilon_{ \pm, x}$. The two occurring biexciton resonances $B_{+-, b} P_{-}^{*}$ associated with $P_{+}$and $\sigma_{+}$circularly polarized light and $B_{+-, b} P_{+}^{*}$ associated with $P_{-}$and $\sigma_{-}$circularly polarized light are energetically degenerate.

The smaller biexciton-binding energy $\epsilon_{b}=\epsilon_{x,+}+$ $\epsilon_{x,-}-\epsilon_{x x,+-, b}$ compared to previous theoretical calculations [100-108] originates from encapsulation of the monolayer of $\mathrm{MoS}_{2}$ in hexagonal boron nitride: Enhanced environmental screening for encapsulated monolayers compared to freestanding samples leads to weaker Coulomb attraction between electrons and holes [109], which reduces the exciton- and biexciton-binding energies for monolayers encapsulated in hexagonal boron nitride [110]. Ultrafast optical wave-mixing experiments $[61,62,111]$ find biexciton-binding energies in the range of the theoretically calculated values, whereas charged biexcitons measured in incoherent photoluminescence spectroscopy have much higher binding energies [112-117].

\section{B. Temporal dynamics}

Since the intensity of the transmitted light $\left|E_{t, \pm}(t)\right|^{2}$ is dominated by the incoming pulse $E_{0, \pm}(t)$ with only slight modifications due to the material response $P_{ \pm}$, the transmission described by Eq. (2) is unsuitable for our following investigations of the details of the nonlinear response. Therefore, we now investigate the nonlinear exciton timedomain dynamics of the experimentally detectable intensity of the reflected light $\left|E_{r,+}(t)\right|^{2} \sim\left|P_{+}\right|^{2}$, which directly monitors the coherent exciton density $\left|P_{+}\right|^{2}$. Throughout this subsection, the pulse area of the input field $E_{0, \pm}(t)$ is kept constant at $\Theta=\int_{-\infty}^{\infty} d t d E_{0, \pm}(t) / \hbar=2 \pi$, where the Pauli blocking effect of a two-level system gives only one complete Rabi flop. Note that an expansion in the manybody hierarchy in Eqs. (4) and (5) is justified only for $\left|P_{ \pm}\right|^{2} \ll 1$ [77]. In this limit, a two-level atom does not show any Rabi oscillations, and, therefore, the used expansion does not apply. In our case, however, oscillations of the density $\left|P_{+}\right|^{2}$ are beyond Pauli blocking and solely due to the exciton-exciton nonlinearities which at the same time ensure $\left|P_{ \pm}\right|^{2} \ll 1$ and even allow for a $2 \pi$ pulse excitation.

At first, we study the mean-field dynamics [described by the first and second lines of Eq. (4)] to understand the nature of the mean-field exciton-exciton interactioninduced nonlinear coherent oscillations. The black curves in Fig. 3(a) represent the intensity of the reflected light (a)

(b)

(c)
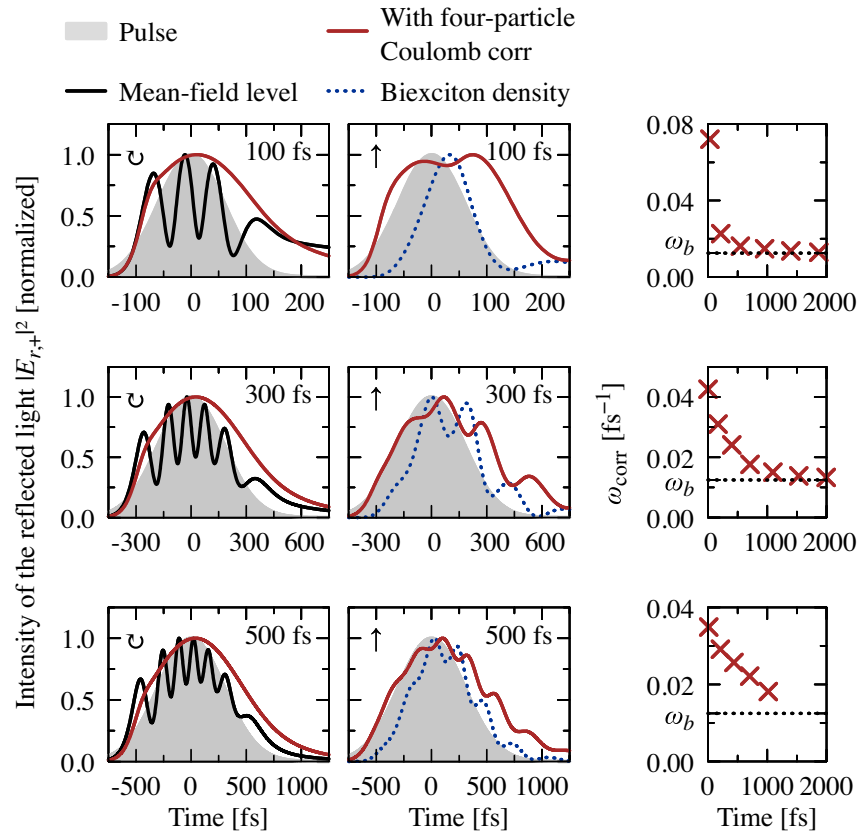

FIG. 3. (a),(b) Normalized intensity of the reflected light $\left|E_{r,+}(t)\right|^{2}$ as a function of time for a monolayer of $\mathrm{MoS}_{2}$ at $5 \mathrm{~K}$. The shaded areas illustrate the exciting (a) circularly (ð) or (b) linearly $(\uparrow)$ polarized pulses with varying intensity FWHM as indicated and fixed pulse areas $\Theta=2 \pi$, and the laser pump energy $\hbar \omega_{p}=\epsilon_{x, \pm}$ is resonant to the exciton resonance energy $\epsilon_{x, \pm}$. The mean-field results and the dynamics including four-particle Coulomb correlations (i.e., biexcitons and two-exciton scattering continua) are shown as black and red solid lines, respectively. The biexciton density $\left|B_{+-, b}\right|^{2}$ is shown as a blue dotted line. (c) Temporal evolution of the oscillation frequency $\omega_{\text {corr }}(t)$ of the intensity of the reflected light $\left|E_{r,+}(t)\right|^{2}$ for linearly polarized excitation including four-particle Coulomb correlations. The frequencies approach the biexciton-binding frequency $\omega_{b}=\epsilon_{b} / \hbar$.

$\left|E_{r,+}(t)\right|^{2}$ in the mean-field limit. Here, the exciton density $\left|P_{+}\right|^{2}$ and, therefore, also the intensity of the reflected light $\left|E_{r,+}(t)\right|^{2}$ almost adiabatically follow the pulse (shaded area) with superimposed oscillations. These mean-fieldinduced oscillations are identical for circularly and linearly polarized excitation, since the dynamics of $P_{+}$and $P_{-}$are decoupled without correlation effects described by the last contribution to Eq. (4). The oscillation frequency was previously analytically approximated for a square pulse $\omega_{\text {mean-field }} \approx 2\left[\left(\epsilon_{g}-\epsilon_{x,+}\right) d^{2} E_{0,+}^{2}\right]^{1 / 3} / \hbar \quad[118]$, where $\epsilon_{g}$ denotes the band gap energy. With increasing pulse duration, the number of oscillations rises, whereas the oscillation frequency $\omega_{\text {mean-field }}$ declines [118-121]. These mean-field-induced density oscillations represent an approximation and are expected to dominate only for weak correlation effects secured if a certain extent of free electron-hole excitations occurs. Consequently, they can be observed for conventional semiconductor quantum wells with low exciton-binding energies $[23,25,26]$. 
However, the mean-field limit does not apply to semiconductors with tightly bound excitons like monolayers of $\mathrm{MoS}_{2}$, which exhibit pronounced excitonic Coulomb correlation effects. This fact restricts the applicability of a pure mean-field theory in the absence of (photo)dopinginduced screening and averaging mechanisms due to free electron-hole pair excitations. In contrast, the inclusion of an efficient coupling of excitons $P_{ \pm}$to biexcitons $B_{+-, b}$ and two-exciton continua $B_{ \pm \pm, \eta \neq b}$ and $B_{+-, \eta \neq b}$ [described by the last line of Eq. (4)] also induces a different exciton dynamics for (i) circularly and (ii) linearly polarized excitation.

(i) The red curves in Fig. 3(a) display a complete breakdown of the mean-field-induced oscillations for circularly (ひ) polarized excitation: Quantum interference of the exciton transition $P_{+}$with the two-exciton scattering continuum $B_{++, \eta \neq b}$ results in excitation-induced dephasing (EID). Thus, the meanfield-induced oscillations vanish. To provide an analytic understanding of the observations, as developed in Appendix B, the exciton dynamics for resonant excitation $\hbar \omega_{p}=\epsilon_{x,+}$ can be approximated by

$\left[\hbar \partial_{t}+\gamma_{0}+\gamma_{r}\right] P_{+}=-i \frac{d E_{0}(t)}{2}-\gamma_{x} P_{+}\left|P_{+}\right|^{2}$.

Compared to the full dynamics described by Eq. (4) and depicted in Fig. 3(a), Pauli blocking is neglected in Eq. (6) [118], and the two-exciton scattering continuum $B_{++, \eta \neq b}$ inducing EID is described by an effective rate $\gamma_{x}$. For dominating EID $\gamma_{x}$, the intensity of the reflected light $\left|E_{r,+}(t)\right|^{2} \sim\left|P_{+}\right|^{2}$ adiabatically follows the pulse $E_{0}(t)$ with the stationary solution $P_{0}$ of Eq. (6) given by (cf. Appendix B)

$$
\left|P_{0}(t)\right|^{2}=\left[\frac{d E_{0}(t)}{2 \gamma_{x}}\right]^{2 / 3} .
$$

(ii) In strong contrast to circularly polarized excitation, the red curves in Fig. 3(b), which characterize the intensity of the reflected light $\left|E_{r,+}(t)\right|^{2}$ for linearly $(\uparrow)$ polarized excitation, again show pronounced nonlinear oscillations. These oscillations are superimposed on the scattering-dominated background which adiabatically follows the pulse. The oscillations occur since linear polarized excitation accesses biexcitons $B_{+-, b}$ in addition to the two-exciton scattering continua $B_{ \pm \pm, \eta \neq b}$ and $B_{+-, \eta \neq b}$. In contrast to the mean-field dynamics, these oscillations show a nontrivial exciton-biexciton excitation exchange with a nonlinear dependence on the time-dependent field amplitude. The excitation exchange can be recognized in the red solid and blue dotted curves in Fig. 3(b), which show that the exciton density $\left|P_{+}\right|^{2} \sim\left|E_{r,+}(t)\right|^{2} \quad$ (red solid curve) exhibits a maximum at minimal biexciton density $\left|B_{+-, \eta=b}\right|^{2}$ (blue dotted curve) and vice versa.
An analytical approximation of the full exciton dynamics yields (cf. Appendix B)

$$
\begin{aligned}
{\left[\hbar \partial_{t}+\gamma_{0}+\gamma_{r}\right] P(t) } & \\
= & -i \frac{d E_{0}(t)}{2}-\gamma_{x} P(t)|P(t)|^{2} \\
& -\frac{W_{+-, b} \hat{W}_{+-, b}}{\hbar} P^{*}(t) \\
& \quad \times \int_{-\infty}^{t} d t^{\prime} e^{-\left(2 \gamma_{0}+i \epsilon_{b}\right) / \hbar\left(t-t^{\prime}\right)}\left[P\left(t^{\prime}\right)\right]^{2} .
\end{aligned}
$$

Because of the linear excitation, Eq. (8) does not distinguish between the different polarizations $P=P_{+}=P_{-}$. Compared to the circular exciton dynamics in Eq. (6), linearly polarized light excites biexcitons described by the last term in Eq. (8). This term is obtained by formal integration of the biexciton dynamics [cf. Eq. (5)], where $\epsilon_{b}=\epsilon_{x,+}+$ $\epsilon_{x,-}-\epsilon_{x x,+-, b}$ denotes the biexciton-binding energy. A perturbative solution of Eq. (8), as performed in Appendix B, leads to oscillations described by $\operatorname{Re}\left[e^{-i \omega_{\text {corr }}(t) t}\right]$ with the approximate time-dependent oscillation frequency $\omega_{\text {corr }}(t)$ :

$$
\begin{aligned}
\omega_{\text {corr }}(t)= & \frac{\epsilon_{b}-i 2 \gamma_{0}}{2 \hbar} \\
& -i \sqrt{-\frac{\left(\epsilon_{b}-i 2 \gamma_{0}\right)^{2}}{4 \hbar^{2}}-\frac{2 W_{+-, b} \hat{W}_{+-, b}}{\hbar^{2}}\left|P_{0}(t)\right|^{2}} .
\end{aligned}
$$

Similar to the discussion above, $\left|P_{0}(t)\right|^{2}$ describes the solution without biexcitons which is dominated by EID and adiabatically follows the pulse; cf. Eq. (7).

The time dependence of the oscillation frequency $\omega_{\text {corr }}(t)$ is plotted in Fig. 3(c). In contrast to our analytical solution in Eq. (9), the numerically evaluated excitation exchange between the exciton and biexciton in Fig. 3(c) includes memory effects introduced by the two-exciton scattering continua. The oscillation frequency $\omega_{\text {corr }}(t)$ is not constant but depends nonlinearly via $\left|P_{0}(t)\right|^{2}$ on the incident light field: The frequency $\omega_{\text {corr }}(t)$ is maximal at the pulse center and decreases afterward. When the driving pulse is gone, the oscillation frequency approaches the constant biexciton frequency $\omega_{\text {corr }}(t) \rightarrow \omega_{b}=$ $\epsilon_{b} / \hbar$. The sophisticated time dependence of the oscillation frequency $\omega_{\text {corr }}(t)$ is beyond an excitonbiexciton beating with a constant biexciton-binding frequency $\omega_{b}$ observed in previous optical wavemixing experiments [122-124]. Since the nonlinear coherent oscillations are determined by the interactions of excitons, biexcitons, and the two-exciton scattering continuum, once measured, the nonlinear coherent oscillations allow one to extract (i) EID $\gamma_{x}$ 
via Eq. (7), (ii) the biexciton-binding energy $\epsilon_{b}$ in the limit of low pulse intensities or after the pulse, where $|P(t)|^{2} \rightarrow 0$ implies $\omega_{\text {corr }}(t) \rightarrow \omega_{b}=\epsilon_{b} / \hbar$ [cf. Eq. (9)], and (iii) the exciton-biexciton interaction strength $W_{+-, b} \hat{W}_{+-, b}$ using Eq. (9) as soon as $\epsilon_{b}$ and $|P(t)|^{2}$ are known.

Compared to conventional semiconductor quantum wells, for instance, based on GaAs, atomically thin semiconductors exhibit more pronounced excitonic nonlinearities with higher biexciton-binding frequencies $\omega_{b}$ and larger exciton-exciton interaction parameters. Therefore, atomically thin semiconductors provide a more pronounced and faster oscillatory modulation of the exciton density during experimentally realizable ultrashort pulses. In contrast, the decreased frequency $\omega_{\text {corr }}(t)$ in conventional semiconductor quantum wells does not suffice to achieve a sufficient number of oscillations during an ultrashort pulse while simultaneously avoiding a considerable excitation of free continuum transitions, which generates additional dephasing. Consequently, the observed nonlinear coherent oscillations in atomically thin semiconductors are in contrast to previous experimental observations of Rabilike oscillations in conventional semiconductor quantum wells [23,25,26]: In the latter case, the simultaneous excitation of free continuum excitations above the band gap obscures pure excitonic nonlinearities. Additionally, weaker Coulomb interactions in conventional semiconductor quantum wells suggest a reduced exciton-biexciton coupling $W_{+-, b} \hat{W}_{+-, b}$ and imply only weak deviations of the oscillation frequency $\omega_{\text {corr }}(t)$ from the biexciton-binding frequency $\omega_{b}$. As a result, the biexciton-binding frequency $\omega_{b}$ is expected to dominate even at high optical excitation powers for semiconductors with weak Coulomb interaction especially after the optical pulse [122-124].

All in all, the observed nonlinear coherent oscillations with frequency $\omega_{\text {corr }}(t)$ originate from Coulomb interactions of excitons with biexcitons and the associated two-exciton scattering continua: The coherent superposition of excitons and biexcitons induces a coherent oscillatory excitation exchange, which is combined with unavoidable decoherence due to quantum interference of excitons with the two-exciton scattering continuum resulting from the same physical process, namely, excitonexciton interactions. This circumstance also demonstrates the different nature of exciton-biexciton superpositions in atomically thin semiconductors compared to Rabi oscillations in quantum dots described by the Pauli blocking nonlinearity [125-128]. Whereas Rabi oscillations in quantum dots are damped by radiative decay [129], phonon-assisted damping [130-133], or off-resonant excitations in the wetting layer $[126,134]$, the exciton density oscillations in atomically thin semiconductors are damped by quantum interference with the two-exciton scattering continuum inducing EID. Therefore, new control parameters enable one to steer the exciton-biexciton oscillations in atomically thin semiconductors by suppressing or enhancing the influence of the damping due to the twoexciton scattering continua.

\section{CONTROL OF NONLINEAR QUANTUM SUPERPOSITIONS}

In the following subsections, we demonstrate that the nonlinear exciton-biexciton excitation exchange can be coherently controlled by a steering of the two-exciton scattering continua employing (Sec. IVA) a laser energy detuning, (Sec. IV B) magnetic fields, and (Sec. IV C) quantum coherent feedback.

\section{A. Laser energy dependence}

The first possibility to modify the coherent optical response is to change the laser energy $\hbar \omega_{p}$ with respect to the exciton energies and, thereby, also with respect to the biexciton and two-exciton scattering continuum. We show that the detuning can be chosen to either amplify or suppress the modulation depths of nonlinear coherent oscillations. This effect originates from the different underlying ratios of excited biexciton and two-exciton scattering continuum densities depending on the laser detuning, where the latter is responsible for EID.

Off-resonant excitation below the exciton energy $\hbar \omega_{p}<$ $\epsilon_{x,+}$ with $\sigma_{+}$circularly polarized light reduces the exciton density $\left|P_{+}\right|^{2}$ and two-exciton scattering continuum densities $\left|B_{++, \eta \neq b}\right|^{2}$ in a similar way. Thus, the intensity of the

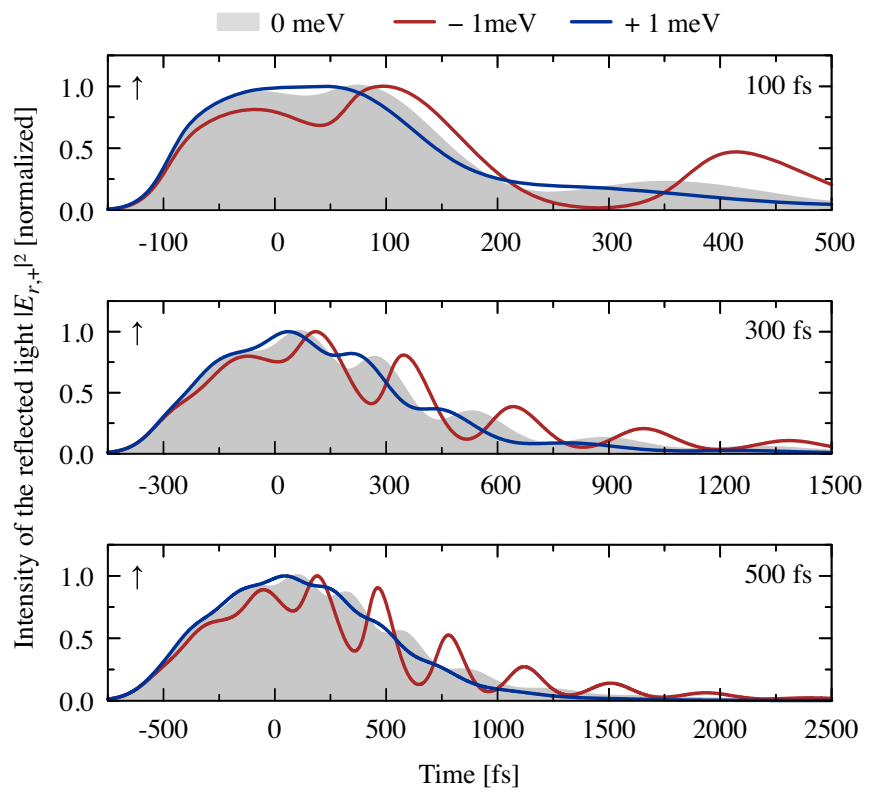

FIG. 4. Normalized time-dependent intensity of the reflected light $\left|E_{r,+}(t)\right|^{2}$ for a monolayer of $\mathrm{MoS}_{2}$ at $5 \mathrm{~K}$ for different detunings of the laser energy $\hbar \omega_{p}$ with respect to the exciton energy $\epsilon_{x,+}: \hbar \omega_{p}-\epsilon_{x,+}=0,-1$, and $+1 \mathrm{meV}$. The intensity FWHM of the different linearly ( $\uparrow)$ polarized pulses with fixed pulse area $\Theta=2 \pi$ is indicated. 


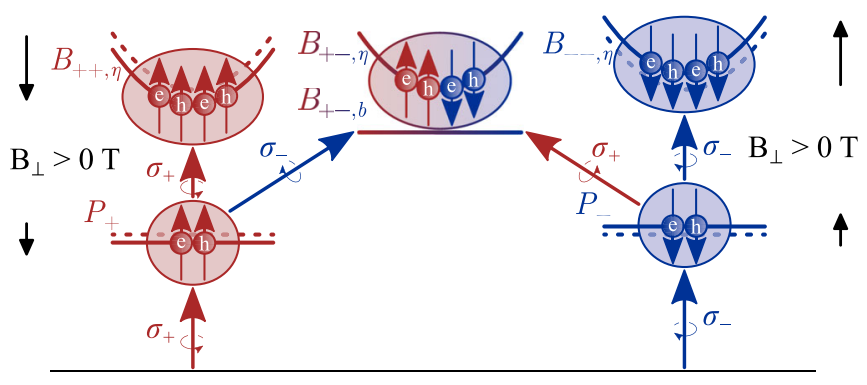

FIG. 5. Illustration of Zeeman shifts of exciton and two-exciton scattering continua in the presence of an out-of-plane magnetic field $\mathrm{B}_{\perp}>0 \mathrm{~T}$; cf. also Fig. 1(b). For $\mathrm{B}_{\perp}>0 \mathrm{~T}$, the exciton transitions $P_{+}$and continua $B_{++, \eta \neq b}$ shift to lower energies, whereas $P_{-}$and $B_{--, \eta \neq b}$ shift to higher energies. The biexciton $B_{+-, b}$ and continua $B_{+-, \eta \neq b}$ experience no shift.

reflected light $\left|E_{r,+}(t)\right|^{2}$ decreases but shows no new qualitative behavior. Therefore, we focus on excitation with linearly polarized light in Fig. 4. Here, the lower laser energy $\hbar \omega_{p}<\epsilon_{x, \pm}$ is closer to the energy $\epsilon_{x x,+-, b}-\epsilon_{x, \mp}$ of the biexciton resonance $B_{+-, \eta} P_{\mp}^{*}$. This closeness enhances the biexciton contributions at the expense of the EID contribution. Consequently, the red curves in Fig. 4 display more pronounced nonlinear coherent oscillations compared to the case with zero detuning shown as gray shaded areas. A laser energy above the exciton energy $\hbar \omega_{p}>\epsilon_{x, \pm}$ is shown as blue curves in Fig. 4. Here, EID increases, and nonlinear coherent oscillations are suppressed due to a lower biexciton contribution.

\section{B. Influence of magnetic-field-induced Zeeman splitting}

In the following, we investigate the nonlinear exciton dynamics in the presence of a magnetic field $\mathrm{B}_{\perp}$, oriented perpendicular to the plane of the atomically thin semiconductor. We show that a magnetic field $\mathrm{B}_{\perp}$ can be used to amplify or suppress the modulation depths of nonlinear coherent oscillations. This opportunity results from the fact that $\mathrm{B}_{\perp}$ induces opposite Zeeman shifts for the exciton transitions $P_{+}$and $P_{-}$[135-146]. The resulting variety of partly competing energy shifts for the biexciton resonance and their continua are illustrated in Fig. 5.

The shifted resonance energies $\epsilon_{x, \pm}$ of the exciton transitions $P_{ \pm}$in Eq. (4) read $\epsilon_{x, \pm} \rightarrow \epsilon_{x} \mp g \mu_{B} \mathrm{~B}_{\perp} / 2$, where $g$ is the exciton $g$ factor and $\mu_{B}$ the Bohr magneton; for details, see Appendix $C$. The energies of the two-exciton scattering continua $B_{ \pm \pm, \eta \neq b}$, driven by two exciton transitions $P_{ \pm} P_{ \pm}$ with the same polarization in Eq. (5), are shifted accordingly: $\epsilon_{x x, \pm \pm, \eta} \rightarrow \epsilon_{x x, \pm \pm, \eta} \mp g \mu_{B} \mathrm{~B}_{\perp}$; cf. Fig. 5. On the other hand, the opposite shifts for $P_{+}$and $P_{-}$cancel each other for the biexciton $B_{+-, \eta=b}$ and the two-exciton scattering continuum $B_{+-, \eta \neq b}$. Thus, their energies $\epsilon_{x x,+-, \eta} \rightarrow$ $\epsilon_{x x,+-, \eta}-g \mu_{B} \mathrm{~B}_{\perp} / 2+g \mu_{B} \mathrm{~B}_{\perp} / 2=\epsilon_{x x,+-, \eta}$ are unchanged. This insight is most important for the possibility to control the interplay of EID and nonlinear coherent oscillations for

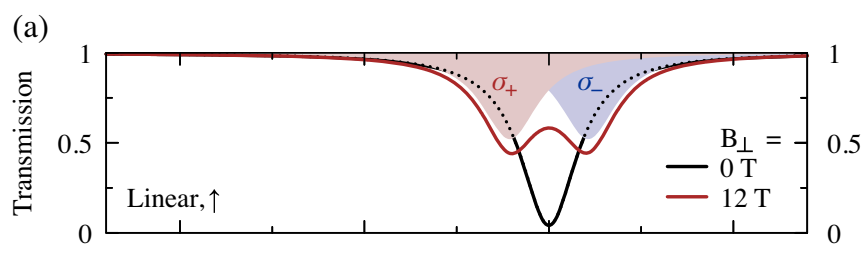

(b)

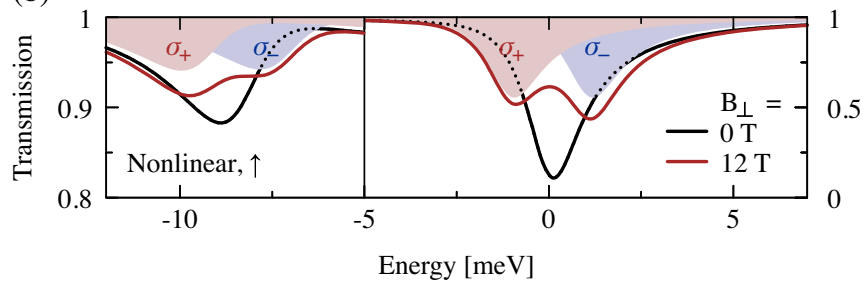

FIG. 6. (a) Linear (pulse area $\Theta \rightarrow 0$ ) and (b) nonlinear (pulse area $\Theta=0.1 \pi$ ) transmission spectra for linearly $(\uparrow)$ polarized excitation with 40 fs pulses (intensity FWHM) for a monolayer of $\mathrm{MoS}_{2}$ at $5 \mathrm{~K}$ for a magnetic field $\mathrm{B}_{\perp}$ of 0 or $12 \mathrm{~T}$. The laser pump energy $\hbar \omega_{p}=\epsilon_{x}$ is resonant to the exciton resonance energy at $\mathrm{B}_{\perp}=0 \mathrm{~T}$. The solid lines represent the linearly polarized transmission and the shaded areas the underlying $\sigma_{+}$and $\sigma_{-}$polarized contributions.

linearly polarized excitation. In particular, the simultaneous excitation of $P_{+}$and $P_{-}$with linearly polarized light inevitably introduces a detuning for at least one of the two exciton transitions $P_{+}$or $P_{-}$. This detuning leads to different excitation ratios of biexcitons $B_{+-, \eta=b}$ and two-exciton scattering continua $B_{++, \eta \neq b}, B_{--, \eta \neq b}$, and $B_{+-, \eta \neq b}$. For clarity and to support this qualitative discussion, we provide the magnetic-field-dependent excitonic Bloch equations in Appendix C.

Before we focus on the time dynamics, we discuss the linear and nonlinear transmission spectra shown in Fig. 6 for different magnetic fields $B_{\perp}$ assuming linearly $(\uparrow)$ polarized excitation: The black curve in Fig. 6(a) shows the transmission spectrum at zero magnetic field $\mathrm{B}_{\perp}=0 \mathrm{~T}$ in the limit of linear optics. For $\mathrm{B}_{\perp}=0 \mathrm{~T}$, the same exciton energies of $P_{+}$and $P_{-}$lead to identical $\sigma_{+}$and $\sigma_{-}$circularly polarized contributions, and the transmission spectrum is characterized by a single Lorentzian exciton resonance. For $\mathrm{B}_{\perp}=12 \mathrm{~T}$, the opposite $\mathrm{B}_{\perp}$-dependent Zeeman shifts for $P_{+}\left(\right.$energy $\left.\epsilon_{x}-g \mu_{B} \mathrm{~B}_{\perp} / 2\right)$ and $P_{-}\left(\right.$energy $\left.\epsilon_{x}+g \mu_{B} \mathrm{~B}_{\perp} / 2\right)$ induce an energy splitting of $g \mu_{B} \mathrm{~B}_{\perp}$, and their energetic degeneracy is lifted. Therefore, the associated $\sigma_{+}$and $\sigma_{-}$ contributions to the transmission, illustrated as shaded areas in Fig. 6(a), are shifted (repelled) with respect to each other. The superposition of $\sigma_{+}$and $\sigma_{-}$contributions determines the total signal and is plotted as a red curve in Fig. 6(a), exhibiting two energetically separated exciton resonances.

The nonlinear transmission spectrum for linearly polarized excitation and $\mathrm{B}_{\perp}=0 \mathrm{~T}$ is shown as a black curve in Fig. 6(b). Here, nonlinear exciton-exciton interactions arising from increased pulse areas lead to EID with asymmetric exciton line shapes and, additionally, a biexciton resonance appears. Again, the two underlying 
$\sigma_{+}$and $\sigma_{-}$circularly polarized contributions to the transmission spectrum are identical for $\mathrm{B}_{\perp}=0 \mathrm{~T}$. This result no longer holds true for $\mathrm{B}_{\perp}=12 \mathrm{~T}$, where the shaded areas in Fig. 6(b) display two energetically separated $\sigma_{+}$and $\sigma_{-}$ contributions, showing exciton resonances with asymmetric line shapes. The two separate contributions also show two biexciton resonances which are nondegenerate due to a magnetic-field-induced splitting of $g \mu_{B} \mathrm{~B}_{\perp}: B_{+-, b} P_{-}^{*}$ with energy $\epsilon_{x x,+-, b}-\epsilon_{x}-g \mu_{B} \mathrm{~B}_{\perp} / 2$ couples to $P_{+}$and $B_{+-, b} P_{+}^{*}$ with energy $\epsilon_{x x,+-, b}-\epsilon_{x}+g \mu_{B} \mathrm{~B}_{\perp} / 2$ couples to $P_{-}$. The red curve in Fig. 6(b) describes the resulting transmission spectrum for $\mathrm{B}_{\perp}=12 \mathrm{~T}$ and linearly polarized excitation. The red curve combines the $\sigma_{+}$and $\sigma_{-}$ contributions illustrated by the shaded areas and reveals two nondegenerate exciton and biexciton resonances.

Next, we focus on the temporal control of excitonexciton interactions by the magnetic field $\mathrm{B}_{\perp}$ and study the temporal dynamics of the intensity of the reflected light $\left|E_{r,+}(t)\right|^{2}$. We briefly discuss the case where we choose circularly polarized excitation with the laser energy $\hbar \omega_{p}=\epsilon_{x}-g \mu_{B} \mathrm{~B}_{\perp} / 2$, i.e., decreasing or increasing with the magnetic field $\mathrm{B}_{\perp}$ to ensure resonant excitation of the exciton transition $P_{+}$. Since the energetic position of the two-exciton scattering continuum $B_{++, \eta \neq b}$ with resonances $B_{++, \eta \neq b} P_{+}^{*}$ at $\epsilon_{x x,++, \eta}-\epsilon_{x}-g \mu_{B} \mathrm{~B}_{\perp} / 2$ decreases to the same extent as the exciton resonance $P_{+}$, the corresponding EID is unaffected by $\mathrm{B}_{\perp}$ (not shown).

In contrast, the magnetic field induces changes for linearly polarized excitation: In Fig. 7, we study only the $\sigma_{+}$circularly polarized component of the intensity of the reflected light $\left|E_{r,+}(t)\right|^{2}$ for excitation with linearly polarized light. The reflected signal is obtained by filtering out $\sigma_{-}$circularly polarized components in the total reflected light field. Again, in Fig. 7, the laser pump energy $\hbar \omega_{p}=\epsilon_{x}-g \mu_{B} \mathrm{~B}_{\perp} / 2$ is varied with the magnetic field to ensure resonant excitation of the exciton transition $P_{+}$. Simultaneous excitation of the exciton transitions $P_{+}$and $P_{-}$by the linearly polarized incident light forms the biexciton $B_{+-, b}$ and two-exciton scattering continuum $B_{+-, \eta \neq b}$.

Because of the opposite Zeeman shifts of $P_{+}$and $P_{-}$, resonant excitation of $P_{+}$inevitably implies off-resonant excitation of $P_{-}$; i.e., $\hbar \omega_{p}=\epsilon_{x}-g \mu_{B} \mathrm{~B}_{\perp} / 2$ implies $\hbar \omega_{p}<$ $\epsilon_{x}+g \mu_{B} \mathrm{~B}_{\perp} / 2$ at $\mathrm{B}_{\perp}>0 \mathrm{~T}$. Therefore, different results are expected for the emission of the filtered reflected intensities $\left|E_{r,+}(t)\right|^{2}$ and $\left|E_{\mathrm{r},-}(t)\right|^{2}$. This implication also suggests a different behavior of the same $\sigma_{+}$circularly polarized component of the corresponding intensity of reflected light $\left|E_{r,+}(t)\right|^{2}$ at magnetic fields with opposite orientations (signs): For $\mathrm{B}_{\perp}>0 \mathrm{~T}$, off-resonant excitation of $P_{-}$ decreases the EID induced by the two-exciton scattering continua $B_{--, \eta \neq b}$ and $B_{+-, \eta \neq b}$ observed in $\left|E_{r,+}(t)\right|^{2}$, whereas the biexciton density $\left|B_{+-, b}\right|^{2}$ increases, because the pump energy $\hbar \omega_{p}$ is energetically closer to the biexciton resonance $B_{+-, b} P_{+}^{*}$. The combination of reduced

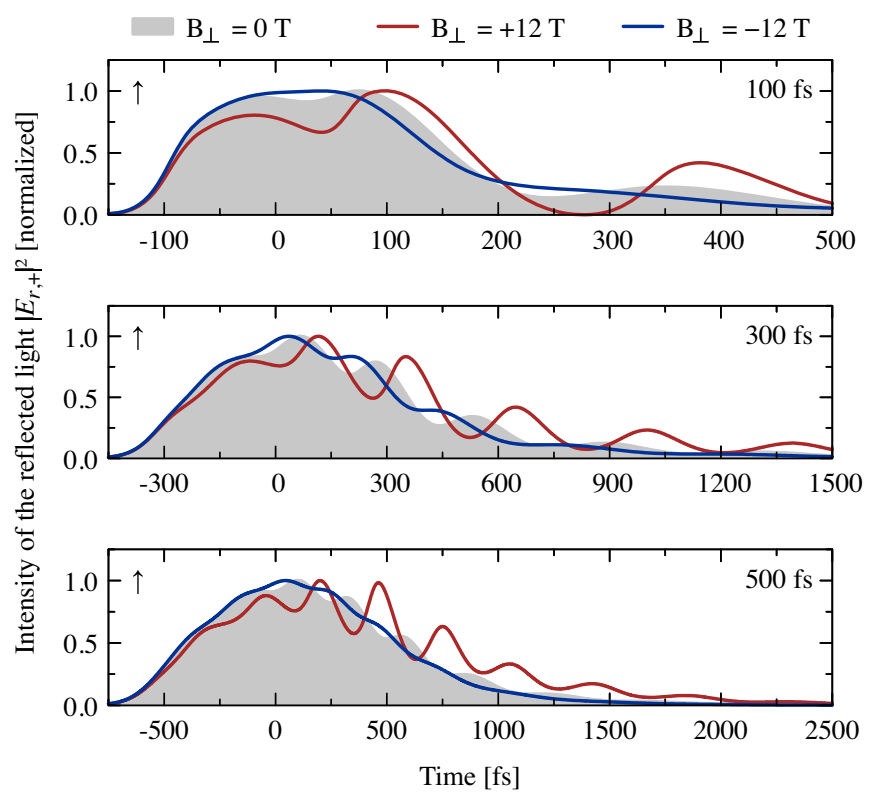

FIG. 7. Normalized time-dependent intensity of the reflected light $\left|E_{r,+}(t)\right|^{2}$ for a monolayer of $\mathrm{MoS}_{2}$ at $5 \mathrm{~K}$ for different magnetic fields $\mathrm{B}_{\perp}$. The intensity FWHM of the different linearly ( $\uparrow$ ) polarized pulses with fixed pulse area $\Theta=2 \pi$ is indicated, and the laser pump energy $\hbar \omega_{p}=\epsilon_{x}-g \mu_{B} \mathrm{~B}_{\perp} / 2$ is varied along with $\mathrm{B}_{\perp}$ to always ensure resonant excitation of the exciton transition $P_{+}$.

EID and an enhanced biexciton density induces a more pronounced nonlinear coherent oscillation for $\mathrm{B}_{\perp}=12 \mathrm{~T}$ plotted as red curves in Fig. 7 compared to $\mathrm{B}_{\perp}=0 \mathrm{~T}$ shown as gray shaded areas. In contrast, the blue lines in Fig. 7 for $B_{\perp}=-12 \mathrm{~T}$ show a lower modulation depth of the nonlinear coherent oscillations, since a laser energy resonant to $P_{+}$implies that the laser energy is above the resonance energy of $P_{-}$; i.e., $\hbar \omega_{p}=\epsilon_{x}-g \mu_{B} \mathrm{~B}_{\perp} / 2$ implies $\hbar \omega_{p}>\epsilon_{x}+g \mu_{B} \mathrm{~B}_{\perp} / 2$ at $\mathrm{B}_{\perp}<0 \mathrm{~T}$. Therefore, the EID observed in $\left|E_{r,+}(t)\right|^{2}$ rises, and a lower biexciton contribution reduces the nonlinear coherent oscillations.

Our results clearly show that coherent nonlinear effects can be controlled by a magnetic field $\mathrm{B}_{\perp}$ perpendicular to the sample. The effect is based on the mutual increase or decrease of the resonance energies of excitons, biexcitons, and two-exciton scattering continua. In particular, this effect enables one to manipulate the quantum interference of excitons with biexcitons and the two-exciton scattering continua and, therefore, allows one to suppress the EID while enhancing the nonlinear coherent oscillations and vice versa.

\section{Coherent feedback control}

A third possibility to control nonlinear coherent oscillations is provided by quantum coherent feedback [147]. We show that feedback, as a polaritonic effect, controls not only the radiative exciton dephasing but, more importantly, 
(a)

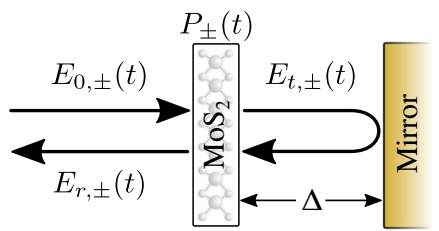

(b)

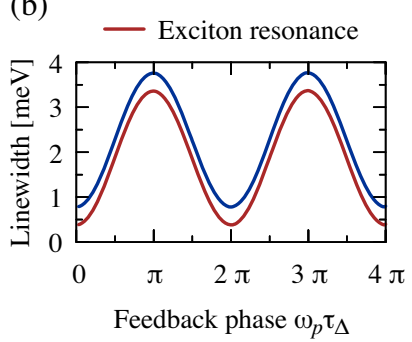

(c)

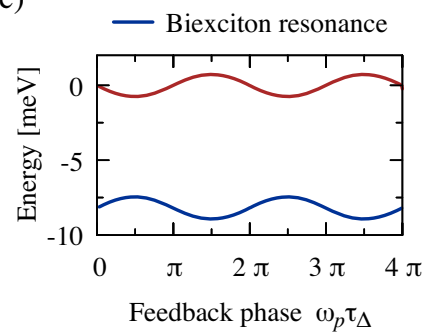

FIG. 8. (a) Illustration of a closed loop with a mirror placed in a distance $\Delta$ from the two-dimensional semiconductor. The incoming light field $E_{0, \pm}(t)$ drives the exciton transitions $P_{ \pm}(t)$. $E_{0, \pm}(t)$ and $P_{ \pm}(t)$ determine the transmitted $E_{t, \pm}(t)$ and reflected $E_{r, \pm}(t)$ light fields. (b),(c) Feedback phase $\omega_{p} \tau_{\Delta}$-dependent (b) linewidths and (c) resonance energies for exciton and biexciton resonances in a monolayer of $\mathrm{MoS}_{2}$ at $5 \mathrm{~K}$ for excitation with low pulse areas $\Theta \rightarrow 0$.

the exciton and biexciton resonance energies and associated oscillator strengths. Thus, feedback can be used to steer the mutual interplay between EID and nonlinear coherent oscillations.

We study a half-sided cavity [148-151] by introducing a reflecting mirror in front of the atomically thin semiconductor as illustrated in Fig. 8(a). These cavities were already experimentally realized but so far studied only for excitation conditions below the onset of nonlinear coherent oscillations [152-158]. The mirror introduces time-delayed feedback of the transmitted light field $E_{t, \pm}(t)$, which enters the Bloch equation (4). $E_{t, \pm}(t)$ is derived using the associated boundary conditions $[52,53]$ :

$$
\begin{aligned}
E_{t, \pm}(t)= & E_{0, \pm}(t)-e^{i \omega_{p} \tau_{\Delta}} E_{0, \pm}\left(t-\tau_{\Delta}\right) \\
& +i \alpha\left[P_{ \pm}^{*}(t)-e^{i \omega_{p} \tau_{\Delta}} P_{ \pm}^{*}\left(t-\tau_{\Delta}\right)\right],
\end{aligned}
$$

with the feedback time $\tau_{\Delta}=\left(n_{r} / c_{0}\right) 2 \Delta$ and the spatial distance $\Delta$ between the atomically thin semiconductor and the mirror; cf. Fig. 8(a).

Before providing numerical evaluations, we present an analytical analysis for short feedback times $\tau_{\Delta}$ compared to the pulse duration. Here, the retarded time arguments in Eq. (10) can be approximately neglected:

$$
E_{t, \pm}(t) \approx\left(1-e^{i \omega_{p} \tau_{\Delta}}\right)\left[E_{0, \pm}(t)+i \alpha P_{ \pm}^{*}(t)\right] .
$$

In this case, the prefactor $\left(1-e^{i \omega_{p} \tau_{\Delta}}\right)$ constitutes the only difference compared to the transmission $E_{t, \pm}(t)$ of the geometry without a mirror; cf. Eq. (2). As derived in detail in Appendix D, Eq. (11) implies that a self-consistent treatment of the light-matter interaction introduces a radiative dephasing $\gamma_{r}\left(\tau_{\Delta}\right)=2 \alpha^{*} d \sin ^{2}\left(\omega_{p} \tau_{\Delta} / 2\right)$ and a radiative energy shift $\delta_{r}\left(\tau_{\Delta}\right)=-\alpha^{*} d \sin \left(\omega_{p} \tau_{\Delta}\right)$ of the transition $P_{ \pm}$depending on the feedback time $\tau_{\Delta}$. The radiative dephasing $\gamma_{r}\left(\tau_{\Delta}\right)$ and radiative energy shift $\delta_{r}\left(\tau_{\Delta}\right)$ originate from the exciton-polariton formation by a coupled excitonlight system: In particular, reradiation of the excitonic polarization density modifies the external light field, while the material properties are unchanged. Therefore, the optical observable belongs to a polariton formed by the coupling of excitons to the light field [159]. However, for convenience, we still use the term exciton even if we actually discuss an exciton-polariton resonance.

The total exciton linewidth including radiative and phonon-mediated contributions $2\left[\gamma_{r}\left(\tau_{\Delta}\right)+\gamma_{0}\right]$ is plotted as a red line in Fig. 8(b). The exciton linewidth is minimal for destructive interference $\omega_{p} \tau_{\Delta}=0,2 \pi, 4 \pi, \ldots$, where the radiative contribution vanishes $2 \gamma_{r}\left(\tau_{\Delta}\right) \rightarrow 0$ and the phonon-mediated contribution of $2 \gamma_{0}=0.4 \mathrm{meV}$ at $5 \mathrm{~K}$ dominates. In contrast, constructive interference $\omega_{p} \tau_{\Delta}=$ $\pi, 3 \pi, \ldots$ leads to the largest radiative linewidths $2 \gamma_{r}\left(\tau_{\Delta}\right)$. The oscillating exciton linewidth is also mirrored in the biexciton resonance $B_{+-, b} P_{\mp}^{*}$ with its linewidth shown by the blue curve in Fig. 8(b). The offset compared to the exciton linewidth is due to assuming a doubled phononmediated contribution to the biexciton resonance linewidth.

The red line in Fig. 8(c) shows the $\omega_{p} \tau_{\Delta}$-dependent sinusoidal oscillations of the radiative exciton energy shift $\delta_{r}\left(\tau_{\Delta}\right)=-\alpha^{*} d \sin \left(\omega_{p} \tau_{\Delta}\right)$. The biexciton resonance energy is plotted as a blue line in Fig. 8(c) and oscillates with a phase difference of $\pi$. The phase difference occurs since a blueshifted $\left(\delta_{r}>0\right.$ ) exciton $P_{ \pm}$(energy $\left.\epsilon_{x, \pm}+\delta_{r}\right)$ yields a redshifted biexciton resonance $B_{+-, \eta} P_{\mp}^{*}$ (energy $\epsilon_{x x,+-b}$, $-\epsilon_{x, \mp}-\delta_{r}$ ) and vice versa. The feedback-dependent energy separation between exciton $P_{ \pm}$and biexciton resonance $B_{+-, \eta} P_{\mp}^{*} \quad$ reads $\quad \epsilon_{b}+2 \delta_{r} \quad$ (with $\quad \epsilon_{b}=\epsilon_{x,+}+\epsilon_{x,-}-$ $\left.\epsilon_{x x,+-, b}\right)$. A decreased exciton-biexciton energy separation $\epsilon_{b}+2 \delta_{r}<\epsilon_{b}$ for $\delta<0$ suggests an increased biexciton oscillator strength. This result can be understood by evaluating the time integral in the last term in Eq. (8), which yields a biexciton oscillator strength inversely proportional to $\epsilon_{b}+$ $2 \delta_{r}$ [160]. Oppositely, $\delta_{r}>0$ implies an increased excitonbiexciton energy separation $\epsilon_{b}+2 \delta_{r}>\epsilon_{b}$, which should decrease the biexciton oscillator strength.

These analytical expectations are verified in the linear reflection spectra $\sum_{ \pm}\left|E_{r, \pm}(\omega)\right|^{2} /\left[\sum_{ \pm}\left|E_{0, \pm}(\omega)\right|^{2}\right]$ in Fig. 9(a) (negligible pulse area $\Theta \rightarrow 0$ ): The red curve in Fig. 9(a) depicts the spectrum for a feedback phase $\omega_{p} \tau_{\Delta}=$ $0.3 \pi$ with a decreased radiative broadening $\left(\gamma_{r}\right)$ and a redshifted exciton resonance $\left(\delta_{r}<0\right)$. The black curve in Fig. 9(a) represents complete constructive interference $\omega_{p} \tau_{\Delta}=\pi$, where the exciton radiative line broadening is maximal and the shift vanishes $\left(\delta_{r}=0\right)$. Increasing the feedback phase to $\omega_{p} \tau_{\Delta}=1.7 \pi$, as illustrated by the blue 


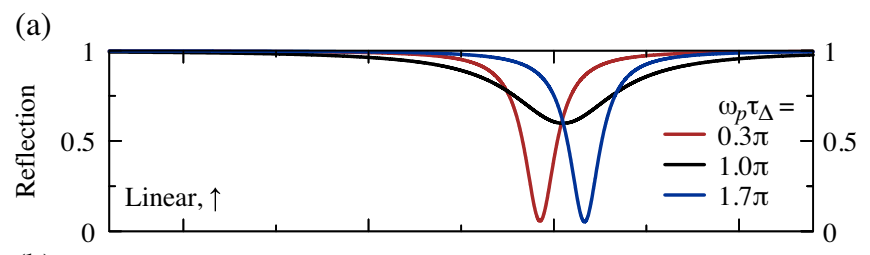

(b)

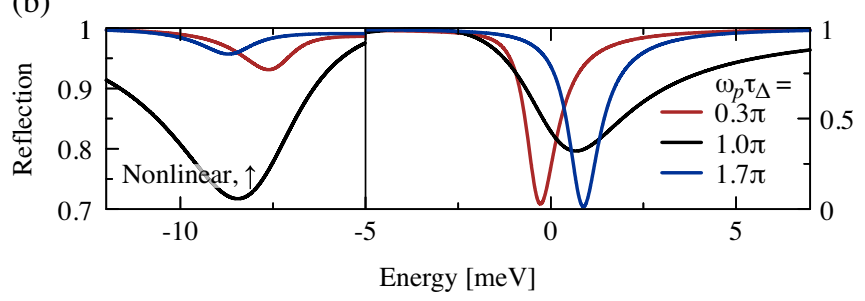

FIG. 9. (a) Linear (pulse area $\Theta \rightarrow 0$ ) and (b) nonlinear (pulse area $\Theta=0.05 \pi$ ) reflection spectra for linearly $(\uparrow)$ polarized excitation with $40 \mathrm{fs}$ pulses (intensity FWHM) for a monolayer of $\mathrm{MoS}_{2}$ at $5 \mathrm{~K}$ for different feedback phases $\omega_{p} \tau_{\Delta}=0.3 \pi, 1.0 \pi$, and $1.7 \pi$. The laser pump energy $\hbar \omega_{p}=\epsilon_{x, \pm}$ is resonant to the exciton resonance energy $\epsilon_{x, \pm}$ at the feedback phase $\omega_{p} \tau_{\Delta}=1.0 \pi$.

curve in Fig. 9(a), decreases the radiative linewidth and the exciton shifts blue $\left(\delta_{r}>0\right)$. Finally, the radiative broadening and shift approach zero for destructive interference $\omega_{p} \tau_{\Delta}=2 \pi$, where the reflection vanishes.

Next, we discuss the influence of quantum coherent feedback on nonlinear exciton-exciton interactions, such as EID and biexciton formation, in the nonlinear reflection spectra. The red curve in Fig. 9(b) represents $\omega_{p} \tau_{\Delta}=0.3 \pi$, where the redshifted exciton $P_{ \pm}\left(\delta_{r}<0\right)$ implies a blueshifted biexciton resonance $B_{+-, \eta} P_{\mp}^{*}$; cf. Fig. 8(c). Perfect constructive interference $\omega_{p} \tau_{\Delta}=\pi$ shown as a black curve in Fig. 9(b) yields an electric field amplification $\left(1-e^{i \omega_{p} \tau_{\Delta}}\right) E_{0, \pm}(t)=2 E_{0, \pm}(t)$ in Eq. (10). The electric field amplification leads to a more pronounced power-dependent exciton line broadening, due to enhanced EID from the twoexciton continuum. At the same time, the biexciton oscillator strength is enhanced.

For the feedback phase $\omega_{p} \tau_{\Delta}=1.7 \pi$, plotted as a blue curve in Fig. 9(b), the exciton line broadening and biexciton oscillator strength are decreased, and the biexciton resonance shifts red. In particular, the comparison of feedback phases $\omega_{p} \tau_{\Delta}=0.3 \pi$ and $1.7 \pi$ shows-as the analytical discussion suggests-a larger biexciton oscillator strength for $\omega_{p} \tau_{\Delta}=0.3 \pi$. Since the biexciton oscillator strength is approximately inversely proportional to the exciton-biexciton energy separation $\epsilon_{b}+2 \delta_{r}, \omega_{p} \tau_{\Delta}=$ $0.3 \pi$ with $\epsilon_{b}+2 \delta_{r}<\epsilon_{b}\left(\delta_{r}<0\right)$ implies a larger oscillator strength than $\omega_{p} \tau_{\Delta}=1.7 \pi$ with $\epsilon_{b}+2 \delta_{r}>\epsilon_{b}\left(\delta_{r}>0\right)$. Importantly, this result demonstrates the possibility to manipulate nonlinear exciton-exciton interactions by feedback. Therefore, a feedback-introducing mirror will most certainly allow one to modify the nonlinear coherent oscillations in the time domain, which is discussed next.
In contrast to the geometry without feedback, the experimentally accessible reflected light $E_{r,+}(t)$ includes also the pure backreflected pulse $-e^{i \omega_{p} \tau_{\Delta}} E_{0,+}\left(t-\tau_{\Delta}\right)$, which obscures the material response $P_{+}$; cf. Eqs. (3) and (10). Therefore, we now examine the reflected light minus the backreflected pulse, which corresponds to the reflection difference $\Delta E_{r,+}(t)$ with and without the atomically thin semiconductor:

$$
\Delta E_{r,+}(t)=E_{r,+}(t)+e^{i \omega_{p} \tau_{\Delta}} E_{0,+}\left(t-\tau_{\Delta}\right) .
$$

Again, we choose laser energies $\hbar \omega_{p}=\epsilon_{x,+}+\delta_{r}$ resonant to the exciton energies.

The red curves in Fig. 10 are obtained for the feedback phase $\omega_{p} \tau_{\Delta}=0.3 \pi$. Here, the effective electric field is approximately decreased by the prefactor $\left(1-e^{i \omega_{p} \tau_{\Delta}}\right)$ in Eq. (11), which naively suggests weaker optical excitation strength-dependent effects compared to the previous results without feedback shown as gray shaded areas in Fig. 10. Strikingly, the opposite holds true for the nonlinear coherent oscillations, and the red curves in Fig. 10 show larger oscillation depths compared to the response without feedback shown as gray shaded areas. The enhancement of the nonlinear coherent oscillations originates from a decreased exciton-biexciton energy separation $\epsilon_{b}+2 \delta_{r}<$ $\epsilon_{b} \quad\left(\delta_{r}<0\right)$ leading to increased biexciton oscillator

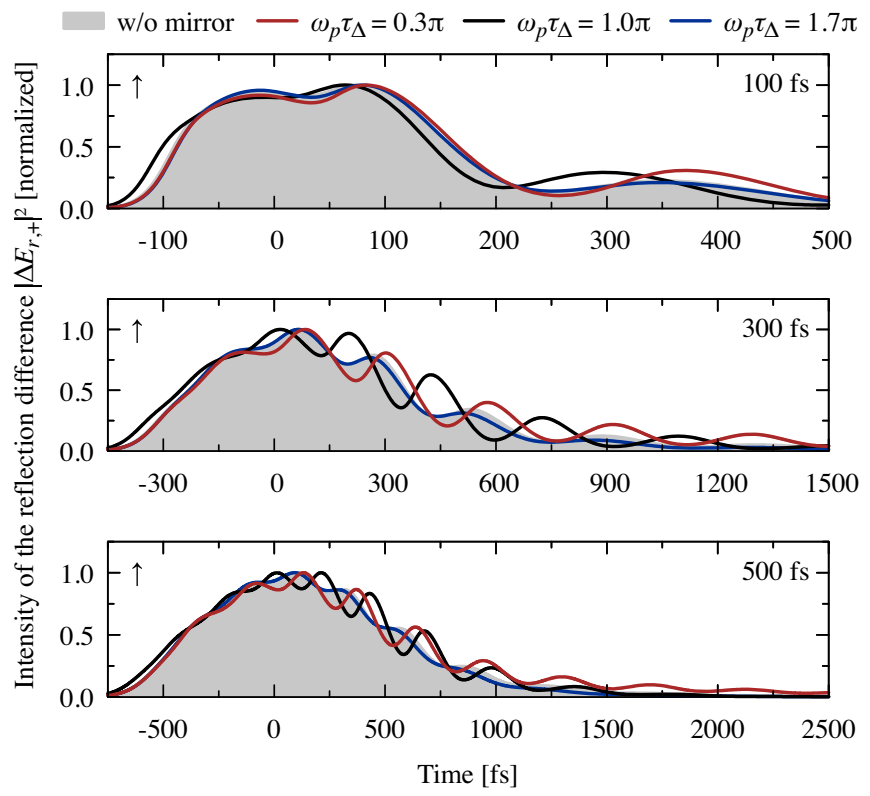

FIG. 10. Normalized time-dependent intensity of the reflection difference $\left|\Delta E_{r,+}(t)\right|^{2}$ for a monolayer of $\mathrm{MoS}_{2}$ at $5 \mathrm{~K}$ for different feedback phases $\omega_{p} \tau_{\Delta}=0.3 \pi, 1.0 \pi$, and $1.7 \pi$. The exciton density in the absence of a mirror is shown as a gray shaded area. The intensity FWHM of the different linearly ( $\uparrow$ ) polarized pulses with fixed pulse area $\Theta=2 \pi$ is indicated. The laser pump energy $\hbar \omega_{p}=\epsilon_{x,+}+\delta_{r}$ is varied along with the detuning $\delta_{r}$ to always ensure resonant excitation of the exciton transition $P_{+}$. 
strengths. Therefore, $\omega_{p} \tau_{\Delta}=0.3 \pi$ combines enhanced nonlinear coherent oscillations with decreased radiative dephasing and EID. The oscillation depths for $\omega_{p} \tau_{\Delta}=$ $0.3 \pi$ are even similar to complete constructive interference $\omega_{p} \tau_{\Delta}=\pi$ shown by the black curves in Fig. 10. However, the enhanced nonlinear coherent oscillations for $\omega_{p} \tau_{\Delta}=\pi$ are counteracted by increased radiative dephasing and EID. For an increasing mirror distance of $\omega_{p} \tau_{\Delta}=1.7 \pi$, plotted as blue lines in Fig. 10, the effectively acting electric field is again decreased. In combination with the increased exciton-biexciton energy separation $\epsilon_{b}+2 \delta_{r}>\epsilon_{b}\left(\delta_{r}>0\right)$ and smaller biexciton oscillator strength, this decrease implies weaker nonlinear coherent oscillations even though the radiative dephasing is suppressed. This behavior is different to the previously studied feedback phase $\omega_{p} \tau_{\Delta}=0.3 \pi$ : $\omega_{p} \tau_{\Delta}=0.3 \pi$ combines enhanced nonlinear coherent oscillations and decreased radiative dephasing and EID, while the oscillations are weakened for $\omega_{p} \tau_{\Delta}=1.7 \pi$.

Our results demonstrate that coherent feedback engineers exciton-exciton interactions by the spatial distance between an atomically thin semiconductor and a mirror. In particular, the biexciton resonance energy and the biexciton oscillator strength can be simultaneously modified. Therefore, the spatial distance between the atomically thin semiconductor and mirror can be adjusted to suppress or enhance nonlinear exciton-biexciton coherent oscillations.

\section{CONCLUSIONS AND PERSPECTIVES}

We report the manifestation of nonlinear excitonbiexciton coherent oscillations activated by exciton-exciton interactions in exciton gases based on optically excited atomically thin semiconductors. The coherent oscillatory exciton-biexciton excitation exchange induces oscillations observed in the intensity of the reflected light with a nonlinear frequency dependence. These oscillations are determined by the mutual interplay of excitons and biexcitons and counteracted by excitation-induced dephasing due to destructive interference of quantum coherence from the two-exciton scattering continua. An experimental realization to measure the proposed nonlinear oscillations requires one to resolve an ultrashort femtosecond timescale, for instance, achievable by taking advantage of high time resolutions realizable by frequency-resolved optical gating [161]. Once measured, the nonlinear oscillations allow one to deduce the excitation-induced dephasing, the biexcitonbinding energy, and the exciton-biexciton interaction strength.

For the coherent manipulation of nonlinear excitonexciton interactions, we propose a number of different control parameters, namely, the polarization of the light field, the pulse detuning, magnetic fields, and quantum coherent feedback. These control parameters pave the way to corresponding experiments by influencing the quantum interference between excitons and biexcitons with the two- exciton scattering continuum, allowing one to enhance or suppress nonlinear coherent oscillations. We expect that the theoretical footing and underlying understanding of nonlinear coherent oscillations is extendable to not only other inorganic and organic semiconductors which enable the preparation of almost pure exciton gases but also other quasibosonic quantum gases with strong Coulomb interactions in condensed matter physics.

\section{ACKNOWLEDGMENTS}

We thank Malte Selig and Dominik Christiansen (TU Berlin), V. Martin Axt (University of Bayreuth), and Tilmann Kuhn (WWU Münster) for stimulating discussions. We gratefully acknowledge support from the Deutsche Forschungsgemeinschaft through Projects No. 420760124 (KN 427/11-1) and No. 163436311-SFB 910 (Project B1) as well as the European Unions Horizon 2020 research and innovation program under Grant Agreement No. 734690 (SONAR). F. K. thanks the Berlin School of Optical Sciences and Quantum Technology.

\section{APPENDIX A: MATERIAL PARAMETERS}

The used material parameters for a monolayer of $\mathrm{MoS}_{2}$ are given in Table I. The numerically evaluated matrix elements which enter the excitonic Bloch equation (4) are listed in Table II for a monolayer of $\mathrm{MoS}_{2}$ encapsulated in

TABLE I. Material parameters for a monolayer of $\mathrm{MoS}_{2}$.

\begin{tabular}{lcc}
\hline \hline Lattice constant & $a_{0}[\mathrm{~nm}]$ & $0.318[162]$ \\
Thickness & $d_{0}[\mathrm{~nm}]$ & $0.626[162]$ \\
Effective electron mass & $m_{e} / m_{0}$ & $0.44[163]$ \\
Effective hole mass & $m_{h} / m_{0}$ & $0.54[163]$ \\
Optical band gap & $\varepsilon_{g}[\mathrm{eV}]$ & $2.48[162]$ \\
In-plane dielectric constant & $\epsilon_{\perp}$ & $12.8[164]$ \\
Plasmon peak energy & $E_{\mathrm{pl}}[\mathrm{eV}]$ & $22.5[164]$ \\
Exciton dephasing at 5 K & $\gamma_{0}[\mathrm{meV}]$ & $0.2[165]$ \\
Effective hopping integral & $t_{0}[\mathrm{eV}]$ & $1.1[55]$ \\
Refractive index of $h$-BN & $n_{r}$ & $\sqrt{4.5}[166]$ \\
\hline \hline
\end{tabular}

TABLE II. Excitonic parameters for a monolayer of $\mathrm{MoS}_{2}$ encapsulated in hexagonal boron nitride.

\begin{tabular}{lcc}
\hline \hline Exciton-binding energy & $\epsilon_{g}-\epsilon_{x, \pm}[\mathrm{eV}]$ & 0.2 \\
Phonon-mediated dephasing at 5 K & $2 \gamma_{0}[\mathrm{meV}]$ & 0.4 \\
Radiative dephasing & $2 \gamma_{\mathrm{r}}[\mathrm{meV}]$ & 1.5 \\
Excitonic dipole matrix element & $d\left[e_{0}\right]$ & 0.1 \\
Pauli blocking parameter & $\hat{d}\left[e_{0} \mathrm{~nm}^{2}\right]$ & 1.3 \\
Coulomb matrix element & $\hat{V}\left[\mathrm{eV} \mathrm{nm}^{2}\right]$ & 1.0 \\
Excitation-induced dephasing & $\gamma_{x}\left[\mathrm{eV} \mathrm{nm}{ }^{2}\right]$ & 0.5 \\
Biexciton-binding energy & $\epsilon_{b}\left[\mathrm{meV}^{2}\right]$ & 8 \\
Exciton-biexciton coupling & $W_{+-, b} \hat{W}_{+-, b}$ & 0.006 \\
& {$\left[\mathrm{eV}^{2} \mathrm{~nm}^{2}\right]$} & \\
\hline
\end{tabular}


hexagonal boron nitride. Analytical approximations for the different matrix elements are discussed in Ref. [50].

\section{APPENDIX B: ANALYTICAL APPROACH}

To provide a better understanding of the temporal behavior, we derive an analytical description of the ultrafast nonlinear exciton density in the following. Since the lightmatter interaction is much weaker compared to excitonexciton scattering, Pauli blocking is neglected $\hat{d}=0$ in Eq. (4). Moreover, excitation-induced shifts are disregarded, i.e., $\hat{V}=0$. In order to describe the exciton dynamics by the Bloch equation [Eq. (4)] solely instead of solving the coupled Maxwell's and excitonic Bloch equations, the radiative dephasing constant $\gamma_{r}$ is explicitly introduced.

(i) The exciton transition $P_{+}$in the rotating frame of the laser frequency $\omega_{p}$ for resonant excitation with circularly polarized light, i.e., $\hbar \omega_{p}=\epsilon_{x,+}$, is determined by

$$
\left[\hbar \partial_{t}+\gamma_{0}+\gamma_{r}\right] P_{+}=-i \frac{d E_{0}(t)}{2}-\gamma_{x} P_{+}\left|P_{+}\right|^{2} .
$$

Here, the two-exciton scattering continuum was solved in a second-order Born-Markov approximation described by an exciton-scattering-induced dephasing rate $\gamma_{x}$ after neglecting energy renormalizations from the two-exciton scattering continuum. Now, we assume that $P_{+}$adiabatically follows the exciting pulse and both the phonon-mediated $\gamma_{0}$ and radiative dephasing $\gamma_{r}$ are small compared to the exciton-scattering-induced dephasing $\gamma_{x}$. Accordingly, the stationary solution $P_{0}(t)$ of Eq. (B1) is given by

$$
P_{0}(t)=-i\left[\frac{d E_{0}(t)}{2 \gamma_{x}}\right]^{1 / 3}
$$

(ii) For linearly polarized excitation, the equations of motion for $P_{+}$and $P_{-}$are identical, which allows one to define $P=P_{+}=P_{-}$. Again, the two-exciton scattering continuum can be solved in a secondorder Born-Markov approximation. This solution introduces exciton-scattering-induced dephasing associated with $\gamma_{x}$, while resulting energy renormalizations are neglected. The following equation of motion results for the resonantly excited exciton transition, i.e., $\hbar \omega_{p}=\epsilon_{x, \pm}$, in the rotating frame $P$ :

$$
\begin{aligned}
& {\left[\hbar \partial_{t}+\gamma_{0}+\gamma_{r}\right] P} \\
& \quad=-i \frac{d E_{0}(t)}{2}-\gamma_{x} P|P|^{2}+i W_{+-, b} B_{+-, b} P^{*} .
\end{aligned}
$$

In addition to the case of circularly polarized excitation [described by Eq. (B1)], Eq. (B3) couples to a single biexciton resonance $B_{+-, b}$ with binding energy $\epsilon_{b}=\epsilon_{x,+}+\epsilon_{x,-}-\epsilon_{x x,+-, \eta=b}$. The biexciton dynamics $B_{+-, b}$ is described by

$$
\left(\hbar \partial_{t}+2 \gamma_{0}+i \epsilon_{b}\right) B_{+-, b}=i \hat{W}_{+-, b} P^{2} .
$$

Formal integration of the biexciton dynamics [Eq. (B4)] and inserting the result in Eq. (B3) gives the following integro-differential equation for the exciton transition $P$ :

$$
\begin{aligned}
{\left[\hbar \partial_{t}+\gamma_{0}+\gamma_{r}\right] P(t) } & \\
=- & i \frac{d E_{0}(t)}{2}-\gamma_{x} P(t)|P(t)|^{2} \\
- & \frac{W_{+-, b} \hat{W}_{+-, b}}{\hbar} P^{*}(t) \\
& \quad \times \int_{-\infty}^{t} d t^{\prime} e^{-\left(2 \gamma_{0}+i \epsilon_{b}\right) / \hbar\left(t-t^{\prime}\right)}\left[P\left(t^{\prime}\right)\right]^{2} .
\end{aligned}
$$

We solve Eq. (B5) in a perturbation approach, where the exciton transition is expanded as follows: $P=P_{0}+\lambda P_{1}+\mathcal{O}\left(\lambda^{2}\right)$, with the ordering parameter $\lambda \ll 1$. The resulting exciton density up to first order in $\lambda$ is given by $|P|^{2}=\left|P_{0}\right|^{2}+\lambda 2 \operatorname{Re}\left[P_{0} P_{1}\right]+\mathcal{O}\left(\lambda^{2}\right)$. Neglecting both the phonon-mediated $\gamma_{0}$ and radiative dephasing $\gamma_{r}$ in a regime where the exciton-scatteringinduced dephasing $\gamma_{x}$ dominates, the zeroth-order $P_{0}(t)$ equation can again be described by a static response; cf. Eq. (B2). Consequently, the first-order equation is left to solve, which reads

$$
\begin{aligned}
\hbar \partial_{t} P_{1}(t) & \\
= & -\gamma_{x}\left\{2\left|P_{0}(t)\right|^{2} P_{1}(t)+\left[P_{0}(t)\right]^{2} P_{1}^{*}(t)\right\} \\
& -\frac{W_{+-, b} \hat{W}_{+-, b}}{2 \gamma_{0}+i \epsilon_{b}}\left[P_{0}(t)\right]^{2} P_{1}^{*}(t) \\
& -\frac{2 W_{+-, b} \hat{W}_{+-, b}}{\hbar}\left|P_{0}(t)\right|^{2} \\
& \times \int_{-\infty}^{t} d t^{\prime} e^{-\left(2 \gamma_{0}+i \epsilon_{b}\right) / \hbar\left(t-t^{\prime}\right)} P_{1}\left(t^{\prime}\right) .
\end{aligned}
$$

The most essential features of Eq. (B6) are approximately captured by the following second-order differential equation:

$$
\partial_{t}^{2} P_{1}+\frac{2 \gamma_{0}+i \epsilon_{b}}{\hbar} \partial_{t} P_{1}+\frac{2 W_{+-, b} \hat{W}_{+-, b}}{\hbar^{2}}\left|P_{0}\right|^{2} P_{1}=0 .
$$

The homogeneous solution of Eq. (B7) is determined by $P_{1} \sim e^{-i \omega_{\text {corr }}(t) t}$ with the oscillation frequency given by 


$$
\begin{aligned}
\omega_{\mathrm{corr}}(t)= & \frac{\epsilon_{b}-i 2 \gamma_{0}}{2 \hbar} \\
& -i \sqrt{-\frac{\left(\epsilon_{b}-i 2 \gamma_{0}\right)^{2}}{4 \hbar^{2}}-\frac{2 W_{+-, b} \hat{W}_{+-, b}}{\hbar^{2}}\left|P_{0}(t)\right|^{2}} .
\end{aligned}
$$

The frequency $\omega_{\text {corr }}(t)$ nonlinearly depends on the zeroth-order solution which adiabatically follows the exciting laser pulse. In the long time regime $t \gg \tau_{\mathrm{FWHM}}$, the frequency approaches the biexcitonbinding frequency $\omega_{\text {corr }}(t) \rightarrow \epsilon_{b} / \hbar$.

\section{APPENDIX C: MAGNETIC FIELD}

An out-of-plane magnetic field $\mathrm{B}_{\perp}$ leads to Zeeman shifts of the conduction and valence bands due to orbital and spin magnetic moments which act with opposite signs at the $K$ and $K^{\prime}$ points [140,167]. The Zeeman shift of $P_{+}$excitons associated with the $K$ valley is given by $g \mu_{B} \mathrm{~B}_{\perp} / 2$, while $-g \mu_{B} \mathrm{~B}_{\perp} / 2$ describes the opposite Zeeman shift of $P_{-}$ excitons in the $K^{\prime}$ valley. The Zeeman shifts $\mp g \mu_{B} \mathrm{~B}_{\perp} / 2$ of $P_{ \pm}$excitons depend on the exciton $g$ factor, the Bohr magneton $\mu_{B}$, and the magnetic field perpendicular to the monolayer sample $\mathrm{B}_{\perp}$. While a $k \cdot p$ perturbation theory accurately determines the $g$ factors of free electrons or holes $[140,168,169]$, the exciton $g$ factors are obtained by taking account of the quasiparticle nature of excitons [170-172]. For our simulations, we use a $g$ factor of excitons of $g=$ -3.06 for a monolayer of $\mathrm{MoS}_{2}$ determined by recent ab initio calculations [171].

The resulting Bloch equations (4) and (5) renormalized by a magnetic field $\mathrm{B}_{\perp}$ read [173]

$$
\begin{aligned}
{\left[\hbar \partial_{t}\right.} & \left.+\gamma_{0}+i\left(\hbar \omega_{p}-\epsilon_{x} \pm g \mu_{B} \mathrm{~B}_{\perp} / 2\right)\right] P_{ \pm} \\
= & -i d E_{t, \pm}^{*}(t)+i \hat{d} E_{t, \pm}^{*}(t)\left|P_{ \pm}\right|^{2}+i \hat{V} P_{ \pm}\left|P_{ \pm}\right|^{2} \\
& +i \sum_{\eta}\left(W_{ \pm \pm, \eta} B_{ \pm \pm, \eta} P_{ \pm}^{*}+W_{+-, \eta} B_{+-, \eta} P_{\mp}^{*}\right) \\
{\left[\hbar \partial_{t}\right.} & \left.+2 \gamma_{0}+i\left(2 \hbar \omega_{p}-\epsilon_{x x, \pm \pm, \eta} \pm g \mu_{B} \mathrm{~B}_{\perp}\right)\right] B_{ \pm \pm, \eta} \\
= & i \hat{W}_{ \pm \pm, \eta} P_{ \pm} P_{ \pm}, \\
{\left[\hbar \partial_{t}\right.} & \left.+2 \gamma_{0}+i\left(2 \hbar \omega_{p}-\epsilon_{x x,+-, \eta}\right)\right] B_{+-, \eta} \\
= & i \hat{W}_{+-, \eta} P_{+} P_{-} .
\end{aligned}
$$

\section{APPENDIX D: ANALYTICAL APPROACH FOR A MIRROR REFLECTION GEOMETRY}

In the following, the radiative dephasing $\gamma_{r}\left(\tau_{\Delta}\right)$ and radiative energy shift $\delta_{r}\left(\tau_{\Delta}\right)$ of the resonance $P_{ \pm}$are derived for an atomically thin semiconductor in the presence of a feedback-introducing mirror. For short feedback times $\tau_{\Delta}$ compared to the pulse duration, the linear transmission spectrum is still accurately reproduced by neglecting the time retardation of the incoming light field $E_{0, \pm}\left(t-\tau_{\Delta}\right) \approx E_{0, \pm}(t)$ and exciton transition $P_{ \pm}\left(t-\tau_{\Delta}\right) \approx$ $P_{ \pm}(t)$; cf. Eq. (11). Inserting the light field at the monolayer position $E_{t, \pm}(t)$ defined in Eq. (11) into the excitonic Bloch equation (4) modifies the excitonic Rabi frequency $\Omega_{ \pm}(t)=$ $d E_{t, \pm}^{*}(t) / \hbar$ valid for short feedback times $\tau_{\Delta}$ :

$$
\Omega_{ \pm}(t) \approx \frac{1}{\hbar} d\left(1-e^{-i \omega_{p} \tau_{\Delta}}\right)\left[E_{0, \pm}^{*}(t)-i \alpha^{*} P_{ \pm}(t)\right] .
$$

Thus, the excitonic Bloch equation for the transition $P_{ \pm}$ restricted to the linear optical response reads

$$
\begin{aligned}
& \left\{\hbar \partial_{t}+\gamma_{r}\left(\tau_{\Delta}\right)+\gamma_{0}+i\left[\hbar \omega_{p}-\epsilon_{x, \pm}-\delta\left(\tau_{\Delta}\right)\right]\right\} P_{ \pm} \\
& \quad=-i d\left(1-e^{-i \omega_{p} \tau_{\Delta}}\right) E_{0, \pm}^{*}(t) .
\end{aligned}
$$

The differential equation (D2) can be analytically solved by Fourier transformation. The resulting frequency-dependent transition $P_{ \pm}(\omega)$ is described by a Lorentzian resonance with a full width at half maximum of $2 \gamma_{r}\left(\tau_{\Delta}\right)+2 \gamma_{0}$ determined by the radiative dephasing $\gamma_{r}\left(\tau_{\Delta}\right)=2 \alpha^{*} d \sin ^{2}\left(\omega_{p} \tau_{\Delta} / 2\right)$ and the phonon-mediated dephasing $\gamma_{0}$. The resonance energy of $P_{ \pm}(\omega)$ is given by $\epsilon_{x, \pm}+\delta\left(\tau_{\Delta}\right)$ including the exciton energy $\epsilon_{x, \pm}$, which is renormalized by the radiative energy shift $\delta_{r}\left(\tau_{\Delta}\right)=-\alpha^{*} d \sin \left(\omega_{p} \tau_{\Delta}\right)$. The radiative dephasing $\gamma_{r}\left(\tau_{\Delta}\right)$ and radiative energy shift $\delta_{r}\left(\tau_{\Delta}\right)$ both originate from reradiation of the excitonic polarization density which modifies the external light field. The mirror modifies only the Rabi frequency defined in Eq. (D1) and no material properties of the atomically thin semiconductor. Therefore, the resulting resonance $P_{ \pm}(\omega)$ describes a polariton formed by the coupling of excitons to the light field.

[1] M. D. Lukin, Colloquium: Trapping and Manipulating Photon States in Atomic Ensembles, Rev. Mod. Phys. 75, 457 (2003).

[2] I. I. Rabi, Space Quantization in a Gyrating Magnetic Field, Phys. Rev. 51, 652 (1937).

[3] L. Allen and J. H. Eberly, Optical Resonance and TwoLevel Atoms (Courier, North Chelmsford, MA, 1987).

[4] H. M. Gibbs, Incoherent Resonance Fluorescence from a Rb Atomic Beam Excited by a Short Coherent Optical Pulse, Phys. Rev. A 8, 446 (1973).

[5] H. Kamada, H. Gotoh, J. Temmyo, T. Takagahara, and H. Ando, Exciton Rabi Oscillation in a Single Quantum Dot, Phys. Rev. Lett. 87, 246401 (2001).

[6] H. Htoon, T. Takagahara, D. Kulik, O. Baklenov, A. L. Holmes, Jr., and C.-K. Shih, Interplay of Rabi Oscillations and Quantum Interference in Semiconductor Quantum Dots, Phys. Rev. Lett. 88, 087401 (2002).

[7] A. Zrenner, E. Beham, S. Stufler, F. Findeis, M. Bichler, and G. Abstreiter, Coherent Properties of a Two-Level 
System Based on a Quantum-Dot Photodiode, Nature (London) 418, 612 (2002).

[8] F. H. L. Koppens, C. Buizert, K.-J. Tielrooij, I. T. Vink, K. C. Nowack, T. Meunier, L.P. Kouwenhoven, and L. M. K. Vandersypen, Driven Coherent Oscillations of a Single Electron Spin in a Quantum Dot, Nature (London) 442, 766 (2006).

[9] J. Danckwerts, K. J. Ahn, J. Förstner, and A. Knorr, Theory of Ultrafast Nonlinear Optics of Coulomb-Coupled Semiconductor Quantum Dots: Rabi Oscillations and PumpProbe Spectra, Phys. Rev. B 73, 165318 (2006).

[10] S. J. Boyle, A. J. Ramsay, A. M. Fox, M. S. Skolnick, A. P. Heberle, and M. Hopkinson, Beating of Exciton-Dressed States in a Single Semiconductor InGaAs/GaAs Quantum Dot, Phys. Rev. Lett. 102, 207401 (2009).

[11] A. J. Ramsay, T. M. Godden, S. J. Boyle, E. M. Gauger, A. Nazir, B. W. Lovett, A. M. Fox, and M. S. Skolnick, Phonon-Induced Rabi-Frequency Renormalization of Optically Driven Single InGaAs/GaAs Quantum Dots, Phys. Rev. Lett. 105, 177402 (2010).

[12] M. Glässl, A. Vagov, S. Lüker, D. E. Reiter, M. D. Croitoru, P. Machnikowski, V. M. Axt, and T. Kuhn, Long-Time Dynamics and Stationary Nonequilibrium of an Optically Driven Strongly Confined Quantum Dot Coupled to Phonons, Phys. Rev. B 84, 195311 (2011).

[13] S. Bounouar, M. Müller, A. M. Barth, M. Glässl, V. M. Axt, and P. Michler, Phonon-Assisted Robust and Deterministic Two-Photon Biexciton Preparation in a Quantum Dot, Phys. Rev. B 91, 161302(R) (2015).

[14] K. Jürgens, F. Lengers, T. Kuhn, and D. E. Reiter, Semiclassical Modeling of Coupled Quantum-Dot-Cavity Systems: From Polaritonlike Dynamics to Rabi Oscillations, Phys. Rev. B 101, 235311 (2020).

[15] T. H. Stievater, X. Li, D. G. Steel, D. Gammon, D. S. Katzer, D. Park, C. Piermarocchi, and L. J. Sham, Rabi Oscillations of Excitons in Single Quantum Dots, Phys. Rev. Lett. 87, 133603 (2001).

[16] D. Press, T. D. Ladd, B. Zhang, and Y. Yamamoto, Complete Quantum Control of a Single Quantum Dot Spin Using Ultrafast Optical Pulses, Nature (London) 456, 218 (2008).

[17] M. Veldhorst, J. C. C. Hwang, C. H. Yang, A. W. Leenstra, B. de Ronde, J. P. Dehollain, J. T. Muhonen, F. E. Hudson, K. M. Itoh, A. Morello, and A. S. Dzurak, An Addressable Quantum Dot Qubit with Fault-Tolerant Control-Fidelity, Nat. Nanotechnol. 9, 981 (2014).

[18] J. Yoneda, K. Takeda, T. Otsuka, T. Nakajima, M. R. Delbecq, G. Allison, T. Honda, T. Kodera, S. Oda, Y. Hoshi, N. Usami, K. M. Itoh, and S. Tarucha, A QuantumDot Spin Qubit with Coherence Limited by Charge Noise and Fidelity Higher than 99.9\%, Nat. Nanotechnol. 13, 102 (2018).

[19] M. Kira and S. W. Koch, Many-Body Correlations and Excitonic Effects in Semiconductor Spectroscopy, Prog. Quantum Electron. 30, 155 (2006).

[20] S. W. Koch, A. Knorr, R. Binder, and M. Lindberg, Microscopic Theory of Rabi Flopping, Photon Echo, and Resonant Pulse Propagation in Semiconductors, Phys. Status Solidi (b) 173, 177 (1992).
[21] S. Glutsch and R. Zimmermann, Coherent Optics for Pumping near the Absorption Edge, Phys. Rev. B 45, 5857 (1992).

[22] T. Kuhn and F. Rossi, Monte Carlo Simulation of Ultrafast Processes in Photoexcited Semiconductors: Coherent and Incoherent Dynamics, Phys. Rev. B 46, 7496 (1992).

[23] S. T. Cundiff, A. Knorr, J. Feldmann, S. W. Koch, E. O. Göbel, and H. Nickel, Rabi Flopping in Semiconductors, Phys. Rev. Lett. 73, 1178 (1994).

[24] C. Fürst, A. Leitenstorfer, A. Nutsch, G. Tränkle, and A. Zrenner, Ultrafast Rabi Oscillations of Free-Carrier Transitions in InP, Phys. Status Solidi (b) 204, 20 (1997).

[25] H. Giessen, A. Knorr, S. Haas, S. W. Koch, S. Linden, J. Kuhl, M. Hetterich, M. Grün, and C. Klingshirn, SelfInduced Transmission on a Free Exciton Resonance in a Semiconductor, Phys. Rev. Lett. 81, 4260 (1998).

[26] A. Schülzgen, R. Binder, M. E. Donovan, M. Lindberg, K. Wundke, H. M. Gibbs, G. Khitrova, and N. Peyghambarian, Direct Observation of Excitonic Rabi Oscillations in Semiconductors, Phys. Rev. Lett. 82, 2346 (1999).

[27] R. Binder and M. Lindberg, Three-Band Excitonic Rabi Oscillations in Semiconductor Quantum Wells, Phys. Rev. B 61, 2830 (2000).

[28] D. McPeake, F. T. Vasko, and E. P. O'Reilly, Rabi Oscillations of Two-Dimensional Electrons under Ultrafast Intersubband Excitation, Phys. Rev. B 68, 193306 (2003).

[29] A. A. Batista and D. S. Citrin, Rabi Flopping in a TwoLevel System with a Time-Dependent Energy Renormalization: Intersubband Transitions in Quantum Wells, Phys. Rev. Lett. 92, 127404 (2004).

[30] C. W. Luo, K. Reimann, M. Woerner, T. Elsaesser, R. Hey, and K. H. Ploog, Phase-Resolved Nonlinear Response of a Two-Dimensional Electron Gas under Femtosecond Intersubband Excitation, Phys. Rev. Lett. 92, 047402 (2004).

[31] K. S. Novoselov, D. Jiang, F. Schedin, T. J. Booth, V. V. Khotkevich, S. V. Morozov, and A. K. Geim, TwoDimensional Atomic Crystals, Proc. Natl. Acad. Sci. U.S.A. 102, 10451 (2005).

[32] A. Splendiani, L. Sun, Y. Zhang, T. Li, J. Kim, C.-Y. Chim, G. Galli, and F. Wang, Emerging Photoluminescence in Monolayer $\mathrm{MoS}_{2}$, Nano Lett. 10, 1271 (2010).

[33] K. F. Mak, C. Lee, J. Hone, J. Shan, and T. F. Heinz, Atomically Thin $\mathrm{MoS}_{2}$ : A New Direct-Gap Semiconductor, Phys. Rev. Lett. 105, 136805 (2010).

[34] T. C. Berkelbach, M. S. Hybertsen, and D. R. Reichman, Theory of Neutral and Charged Excitons in Monolayer Transition Metal Dichalcogenides, Phys. Rev. B 88, 045318 (2013).

[35] D. Y. Qiu, F. H. da Jornada, and S. G. Louie, Optical Spectrum of $\mathrm{MoS}_{2}$ : Many-Body Effects and Diversity of Exciton States, Phys. Rev. Lett. 111, 216805 (2013).

[36] H. M. Hill, A. F. Rigosi, C. Roquelet, A. Chernikov, T. C. Berkelbach, D. R. Reichman, M. S. Hybertsen, L. E. Brus, and T. F. Heinz, Observation of Excitonic Rydberg States in Monolayer $\mathrm{MoS}_{2}$ and $\mathrm{WS}_{2}$ by Photoluminescence Excitation Spectroscopy, Nano Lett. 15, 2992 (2015).

[37] T. Deilmann and K.S. Thygesen, Finite-Momentum Exciton Landscape in Mono- and Bilayer Transition Metal Dichalcogenides, 2D Mater. 6, 035003 (2019). 
[38] M. M. Glazov, T. Amand, X. Marie, D. Lagarde, L. Bouet, and B. Urbaszek, Exciton Fine Structure and Spin Decoherence in Monolayers of Transition Metal Dichalcogenides, Phys. Rev. B 89, 201302(R) (2014).

[39] M. M. Glazov, E. L. Ivchenko, G. Wang, T. Amand, X. Marie, B. Urbaszek, and B. L. Liu, Spin and Valley Dynamics of Excitons in Transition Metal Dichalcogenide Monolayers, Phys. Status Solidi (b) 252, 2349 (2015).

[40] A. Steinhoff, J.-H. Kim, F. Jahnke, M. Rösner, D.-S. Kim, C. Lee, G. H. Han, M. S. Jeong, T. O. Wehling, and C. Gies, Efficient Excitonic Photoluminescence in Direct and Indirect Band Gap Monolayer $\mathrm{MoS}_{2}$, Nano Lett. 15, 6841 (2015).

[41] R. Schmidt, G. Berghäuser, R. Schneider, M. Selig, P. Tonndorf, E. Malić, A. Knorr, S. M. de Vasconcellos, and R. Bratschitsch, Ultrafast Coulomb-Induced Intervalley Coupling in Atomically Thin $\mathrm{WS}_{2}$, Nano Lett. 16, 2945 (2016).

[42] C. Robert, M. A. Semina, F. Cadiz, M. Manca, E. Courtade, T. Taniguchi, K. Watanabe, H. Cai, S. Tongay, B. Lassagne, P. Renucci, T. Amand, X. Marie, M. M. Glazov, and B. Urbaszek, Optical Spectroscopy of Excited Exciton States in $\mathrm{MoS}_{2}$ Monolayers in van der Waals Heterostructures, Phys. Rev. Mater. 2, 011001 (2018).

[43] B. Han, C. Robert, E. Courtade, M. Manca, S. Shree, T. Amand, P. Renucci, T. Taniguchi, K. Watanabe, X. Marie, L. E. Golub, M. M. Glazov, and B. Urbaszek, Exciton States in Monolayer $\mathrm{MoSe}_{2}$ and $\mathrm{MoTe}_{2}$ Probed by Upconversion Spectroscopy, Phys. Rev. X 8, 031073 (2018).

[44] G. Moody, C. K. Dass, K. Hao, C.-H. Chen, L.-J. Li, A. Singh, K. Tran, G. Clark, X. Xu, G. Berghäuser, E. Malić, A. Knorr, and X. Li, Intrinsic Homogeneous Linewidth and Broadening Mechanisms of Excitons in Monolayer Transition Metal Dichalcogenides, Nat. Commun. 6, 8315 (2015).

[45] F. Katsch, M. Selig, and A. Knorr, Exciton-ScatteringInduced Dephasing in Two-Dimensional Semiconductors, Phys. Rev. Lett. 124, 257402 (2020).

[46] A. L. Ivanov and H. Haug, Self-Consistent Theory of the Biexciton Optical Nonlinearity, Phys. Rev. B 48, 1490 (1993).

[47] F. Katsch, M. Selig, A. Carmele, and A. Knorr, Theory of Exciton-Exciton Interactions in Monolayer Transition Metal Dichalcogenides, Phys. Status Solidi (b) 255, 1800185 (2018).

[48] V. M. Axt, K. Victor, and T. Kuhn, The Exciton-Exciton Continuum and Its Contribution to Four-Wave Mixing Signals, Phys. Status Solidi (b) 206, 189 (1998).

[49] W. Schäfer and M. Wegener, Semiconductor Optics and Transport Phenomena (Springer Science, New York, 2013).

[50] R. Takayama, N. H. Kwong, I. Rumyantsev, M. KuwataGonokami, and R. Binder, T-Matrix Analysis of Biexcitonic Correlations in the Nonlinear Optical Response of Semiconductor Quantum Wells, Eur. Phys. J. B 25, 445 (2002).

[51] V. M. Axt and A. Stahl, A Dynamics-Controlled Truncation Scheme for the Hierarchy of Density Matrices in Semiconductor Optics, Z. Phys. B 93, 195 (1994).
[52] A. Knorr, S. Hughes, T. Stroucken, and S. W. Koch, Theory of Ultrafast Spatio-temporal Dynamics in Semiconductor Heterostructures, Chem. Phys. 210, 27 (1996).

[53] T. Stroucken, A. Knorr, P. Thomas, and S. W. Koch, Coherent Dynamics of Radiatively Coupled Quantum-Well Excitons, Phys. Rev. B 53, 2026 (1996).

[54] F. Katsch, M. Selig, and A. Knorr, Theory of Coherent Pump-Probe Spectroscopy in Monolayer Transition Metal Dichalcogenides, 2D Mater. 7, 015021 (2020).

[55] D. Xiao, G.-B. Liu, W. Feng, X. Xu, and W. Yao, Coupled Spin and Valley Physics in Monolayers of $\mathrm{MoS}_{2}$ and Other Group-VI Dichalcogenides, Phys. Rev. Lett. 108, 196802 (2012).

[56] M. Selig, G. Berghäuser, A. Raja, P. Nagler, C. Schüller, T. F. Heinz, T. Korn, A. Chernikov, E. Malić, and A. Knorr, Excitonic Linewidth and Coherence Lifetime in Monolayer Transition Metal Dichalcogenides, Nat. Commun. 7, 13279 (2016).

[57] Z. Khatibi, M. Feierabend, M. Selig, S. Brem, C. Linderälv, P. Erhart, and E. Malić, Impact of Strain on the Excitonic Linewidth in Transition Metal Dichalcogenides, 2D Mater. 6, 015015 (2019).

[58] F. Lengers, T. Kuhn, and D. E. Reiter, Theory of the Absorption Line Shape in Monolayers of Transition Metal Dichalcogenides, Phys. Rev. B 101, 155304 (2020).

[59] M. Selig, F. Katsch, R. Schmidt, S. M. de Vasconcellos, R. Bratschitsch, E. Malić, and A. Knorr, Ultrafast Dynamics in Monolayer Transition Metal Dichalcogenides: Interplay of Dark Excitons, Phonons, and Intervalley Exchange, Phys. Rev. Research 1, 022007(R) (2019).

[60] E. J. Sie, A. J. Frenzel, Y.-. Lee, J. Kong, and N. Gedik, Intervalley Biexcitons and Many-Body Effects in Monolayer $\mathrm{MoS}_{2}$, Phys. Rev. B 92, 125417 (2015).

[61] K. Hao, J. F. Specht, P. Nagler, L. Xu, K. Tran, A. Singh, C. K. Dass, C. Schüller, T. Korn, M. Richter, A. Knorr, X. $\mathrm{Li}$, and G. Moody, Neutral and Charged Inter-valley Biexcitons in Monolayer $\mathrm{MoSe}_{2}$, Nat. Commun. 8, 15552 (2017).

[62] A. Steinhoff, M. Florian, A. Singh, K. Tran, M. Kolarczik, S. Helmrich, A. W. Achtstein, U. Woggon, N. Owschimikow, F. Jahnke, and X. Li, Biexciton Fine Structure in Monolayer Transition Metal Dichalcogenides, Nat. Phys. 14, 1199 (2018).

[63] G. Bartels, A. Stahl, V. M. Axt, B. Haase, U. Neukirch, and J. Gutowski, Identification of Higher-Order Electronic Coherences in Semiconductors by Their Signature in Four-Wave-Mixing Signals, Phys. Rev. Lett. 81, 5880 (1998).

[64] E. Finger, S. Kraft, M. Hofmann, T. Meier, S. W. Koch, W. Stolz, W. W. Rühle, and A. Wieck, Coulomb Correlations and Biexciton Signatures in Coherent Excitation Spectroscopy of Semiconductor Quantum Wells, Phys. Status Solidi (b) 234, 424 (2002).

[65] T. Voss, H. G. Breunig, I. Rückmann, J. Gutowski, V. M. Axt, and T. Kuhn, Biexcitonic Effects in the Coherent Control of the Excitonic Polarization Detected in SixWave-Mixing Signals, Phys. Rev. B 66, 155301 (2002).

[66] I. Kuznetsova, P. Thomas, T. Meier, T. Zhang, X. Li, R. P. Mirin, and S. T. Cundiff, Signatures of Many-Particle Correlations in Two-Dimensional Fourier-Transform 
Spectra of Semiconductor Nanostructures, Solid State Commun. 142, 154 (2007).

[67] S. Schumacher, G. Czycholl, F. Jahnke, I. Kudyk, L. Wischmeier, I. Rückmann, T. Voss, J. Gutowski, A. Gust, and D. Hommel, Coherent Propagation of Polaritons in Semiconductor Heterostructures: Nonlinear Pulse Transmission in Theory and Experiment, Phys. Rev. B 72, 081308(R) (2005).

[68] S. Schumacher, G. Czycholl, and F. Jahnke, Coherent Propagation of Polaritons in the Nonlinear Optical Regime, Phys. Rev. B 73, 035318 (2006).

[69] N. H. Kwong, R. Takayama, I. Rumyantsev, M. KuwataGonokami, and R. Binder, Third-Order Exciton-Correlation and Nonlinear Cavity-Polariton Effects in Semiconductor Microcavities, Phys. Rev. B 64, 045316 (2001).

[70] S. Savasta, O. Di Stefano, and R. Girlanda, Many-Body and Correlation Effects on Parametric Polariton Amplification in Semiconductor Microcavities, Phys. Rev. Lett. 90, 096403 (2003).

[71] S. Schumacher, N. H. Kwong, and R. Binder, Influence of Exciton-Exciton Correlations on the Polarization Characteristics of Polariton Amplification in Semiconductor Microcavities, Phys. Rev. B 76, 245324 (2007).

[72] R. Oszwałdowski, M. Reichelt, T. Meier, S. W. Koch, and M. Rohlfing, Nonlinear Optical Response of the Si(111)$(2 \times 1)$ Surface Exciton: Influence of Biexciton Many-Body Correlations, Phys. Rev. B 71, 235324 (2005).

[73] T. Meier, P. Thomas, and S. W. Koch, Linear and Nonlinear Optical Properties of Semiconductor Nanorings with Magnetic Field and Disorder-Influence on Excitons and Biexcitons, Eur. Phys. J. B 22, 249 (2001).

[74] T. Meier, C. Sieh, E. Finger, W. Stolz, W. W. Rühle, P. Thomas, S. W. Koch, and A. D. Wieck, Signatures of Biexcitons and Triexcitons in Coherent Non-degenerate Semiconductor Optics, Phys. Status Solidi (b) 238, 537 (2003).

[75] B. Haase, U. Neukirch, J. Meinertz, J. Gutowski, V. M. Axt, G. Bartels, A. Stahl, J. Nürnberger, and W. Faschinger, Intensity Dependence of Signals Obtained in Four-WaveMixing Geometry: Influence of Higher-Order Contributions, J. Cryst. Growth 214, 852 (2000).

[76] M. Buck, L. Wischmeier, S. Schumacher, G. Czycholl, F. Jahnke, T. Voss, I. Rückmann, and J. Gutowski, LightPolarization and Intensity Dependence of Higher-Order Nonlinearities in Excitonic FWM Signals, Eur. Phys. J. B 42, 175 (2004).

[77] A. Steinhoff, M. Florian, M. Rösner, G. Schönhoff, T. O. Wehling, and F. Jahnke, Exciton Fission in Monolayer Transition Metal Dichalcogenide Semiconductors, Nat. Commun. 8, 1166 (2017).

[78] K. F. Mak, K. He, C. Lee, G. H. Lee, J. Hone, T. F. Heinz, and J. Shan, Tightly Bound Trions in Monolayer $\mathrm{MoS}_{2}$, Nat. Mater. 12, 207 (2013).

[79] J. D. Lin, C. Han, F. Wang, R. Wang, D. Xiang, S. Qin, X.-A. Zhang, L. Wang, H. Zhang, A. T. S. Wee, and W. Chen, Electron-Doping-Enhanced Trion Formation in Monolayer Molybdenum Disulfide Functionalized with Cesium Carbonate, ACS Nano 8, 5323 (2014).

[80] Y. Li, Z. Qi, M. Liu, Y. Wang, X. Cheng, G. Zhang, and L. Sheng, Photoluminescence of Monolayer $\mathrm{MoS}_{2}$ on
$\mathrm{LaAlO}_{3}$ and $\mathrm{SrTiO}_{3}$ Substrates, Nanoscale 6, 15248 (2014).

[81] P. K. Chow, R. B. Jacobs-Gedrim, J. Gao, T.-M. Lu, B. Yu, H. Terrones, and N. Koratkar, Defect-Induced Photoluminescence in Monolayer Semiconducting Transition Metal Dichalcogenides, ACS Nano 9, 1520 (2015).

[82] M. Drüppel, T. Deilmann, P. Krüger, and M. Rohlfing, Diversity of Trion States and Substrate Effects in the Optical Properties of an $\mathrm{MoS}_{2}$ Monolayer, Nat. Commun. 8, 2117 (2017).

[83] T. Deilmann and K. S. Thygesen, Dark Excitations in Monolayer Transition Metal Dichalcogenides, Phys. Rev. B 96, 201113(R) (2017).

[84] M. Florian, M. Hartmann, A. Steinhoff, J. Klein, A. W. Holleitner, J. J. Finley, T. O. Wehling, M. Kaniber, and C. Gies, The Dielectric Impact of Layer Distances on Exciton and Trion Binding Energies in van der Waals Heterostructures, Nano Lett. 18, 2725 (2018).

[85] D. Vaclavkova, J. Wyzula, K. Nogajewski, M. Bartos, A. O. Slobodeniuk, C. Faugeras, M. Potemski, and M. R. Molas, Singlet and Triplet Trions in $\mathrm{WS}_{2}$ Monolayer Encapsulated in Hexagonal Boron Nitride, Nanotechnology 29, 325705 (2018).

[86] A. Arora, T. Deilmann, T. Reichenauer, J. Kern, S. M. de Vasconcellos, M. Rohlfing, and R. Bratschitsch, Excited-State Trions in Monolayer $\mathrm{WS}_{2}$, Phys. Rev. Lett. 123, 167401 (2019).

[87] R. Tempelaar and T. C. Berkelbach, Many-Body Simulation of Two-Dimensional Electronic Spectroscopy of Excitons and Trions in Monolayer Transition Metal Dichalcogenides, Nat. Commun. 10, 3419 (2019).

[88] A. Arora, N. K. Wessling, T. Deilmann, T. Reichenauer, P. Steeger, P. Kossacki, M. Potemski, S. M. de Vasconcellos, M. Rohlfing, and R. Bratschitsch, Dark Trions Govern the Temperature-Dependent Optical Absorption and Emission of Doped Atomically Thin Semiconductors, Phys. Rev. B 101, 241413(R) (2020).

[89] A. Singh, G. Moody, K. Tran, M. E. Scott, V. Overbeck, G. Berghäuser, J. Schaibley, E. J. Seifert, D. Pleskot, N. M. Gabor, J. Yan, D. G. Mandrus, M. Richter, E. Malić, X. Xu, and X. Li, Trion Formation Dynamics in Monolayer Transition Metal Dichalcogenides, Phys. Rev. B 93, 041401(R) (2016).

[90] C. Robert, D. Lagarde, F. Cadiz, G. Wang, B. Lassagne, T. Amand, A. Balocchi, P. Renucci, S. Tongay, B. Urbaszek, and X. Marie, Exciton Radiative Lifetime in Transition Metal Dichalcogenide Monolayers, Phys. Rev. B 93, 205423 (2016).

[91] T. Godde, D. Schmidt, J. Schmutzler, M. Aßmann, J. Debus, F. Withers, E. M. Alexeev, O. Del Pozo-Zamudio, O. V. Skrypka, K. S. Novoselov, M. Bayer, and A. I. Tartakovskii, Exciton and Trion Dynamics in Atomically Thin $\mathrm{MoSe}_{2}$ and $\mathrm{WSe}_{2}$ : Effect of Localization, Phys. Rev. B 94, 165301 (2016).

[92] J. S. Ross, S. Wu, H. Yu, N. J. Ghimire, A. M. Jones, G. Aivazian, J. Yan, D. G. Mandrus, D. Xiao, Q. Yao, and X. $\mathrm{Xu}$, Electrical Control of Neutral and Charged Excitons in a Monolayer Semiconductor, Nat. Commun. 4, 1474 (2013).

[93] S.-Y. Chen, T. Goldstein, T. Taniguchi, K. Watanabe, and J. Yan, Coulomb-Bound Four- and Five-Particle Intervalley 
States in an Atomically-Thin Semiconductor, Nat. Commun. 9, 3717 (2018).

[94] Z. Ye, L. Waldecker, E. Y. Ma, D. Rhodes, A. Antony, B. Kim, X.-X. Zhang, M. Deng, Y. Jiang, Z. Lu, D. Smirnov, K. Watanabe, T. Taniguchi, J. Hone, and T. F. Heinz, Efficient Generation of Neutral and Charged Biexcitons in Encapsulated $\mathrm{WSe}_{2}$ Monolayers, Nat. Commun. 9, 3718 (2018).

[95] Z. Li, T. Wang, Z. Lu, C. Jin, Y. Chen, Y. Meng, Z. Lian, T. Taniguchi, K. Watanabe, S. Zhang, D. Smirnov, and S.-F. Shi, Revealing the Biexciton and Trion-Exciton Complexes in BN Encapsulated $\mathrm{WSe}_{2}$, Nat. Commun. 9, 3719 (2018).

[96] M. Barbone, A. R.-P. Montblanch, D. M. Kara, C. Palacios-Berraquero, A. R. Cadore, D. De Fazio, B. Pingault, E. Mostaani, H. Li, B. Chen, K. Watanabe, T. Taniguchi, S. Tongay, G. Wang, A. C. Ferrari, and M. Atatüre, ChargeTuneable Biexciton Complexes in Monolayer $\mathrm{WSe}_{2}$, Nat. Commun. 9, 3721 (2018).

[97] Z. Li, T. Wang, C. Jin, Z. Lu, Z. Lian, Y. Meng, M. Blei, M. Gao, T. Taniguchi, K. Watanabe, T. Ren, T. Cao, S. Tongay, D. Smirnov, L. Zhang, and S.-F. Shi, Momentum-Dark Intervalley Exciton in Monolayer Tungsten Diselenide Brightened via Chiral Phonon, ACS Nano 13, 14107 (2019).

[98] M. Paur, A. J. Molina-Mendoza, R. Bratschitsch, K. Watanabe, T. Taniguchi, and T. Mueller, Electroluminescence from Multi-particle Exciton Complexes in Transition Metal Dichalcogenide Semiconductors, Nat. Commun. 10, 1709 (2019).

[99] F. Cadiz, E. Courtade, C. Robert, G. Wang, Y. Shen, H. Cai, T. Taniguchi, K. Watanabe, H. Carrere, D. Lagarde, M. Manca, T. Amand, P. Renucci, S. Tongay, X. Marie, and B. Urbaszek, Excitonic Linewidth Approaching the Homogeneous Limit in $\mathrm{MoS}_{2}$-Based van der Waals Heterostructures, Phys. Rev. X 7, 021026 (2017).

[100] D. K. Zhang, D. W. Kidd, and K. Varga, Excited Biexcitons in Transition Metal Dichalcogenides, Nano Lett. 15, 7002 (2015).

[101] M. Z. Mayers, T. C. Berkelbach, M. S. Hybertsen, and D. R. Reichman, Binding Energies and Spatial Structures of Small Carrier Complexes in Monolayer TransitionMetal Dichalcogenides via Diffusion Monte Carlo, Phys. Rev. B 92, 161404(R) (2015).

[102] D. W. Kidd, D. K. Zhang, and K. Varga, Binding Energies and Structures of Two-Dimensional Excitonic Complexes in Transition Metal Dichalcogenides, Phys. Rev. B 93, 125423 (2016).

[103] E. Mostaani, M. Szyniszewski, C. H. Price, R. Maezono, M. Danovich, R. J. Hunt, N. D. Drummond, and V. I. Fal'ko, Diffusion Quantum Monte Carlo Study of Excitonic Complexes in Two-Dimensional Transition-Metal Dichalcogenides, Phys. Rev. B 96, 075431 (2017).

[104] R. Y. Kezerashvili and S. M. Tsiklauri, Trion and Biexciton in Monolayer Transition Metal Dichalcogenides, FewBody Syst. 58, 18 (2017).

[105] M. Szyniszewski, E. Mostaani, N. D. Drummond, and V. I. Fal'ko, Binding Energies of Trions and Biexcitons in TwoDimensional Semiconductors from Diffusion Quantum Monte Carlo Calculations, Phys. Rev. B 95, 081301(R) (2017).
[106] M. Van der Donck, M. Zarenia, and F. M. Peeters, Excitons, Trions, and Biexcitons in Transition-Metal Dichalcogenides: Magnetic-Field Dependence, Phys. Rev. B 97, 195408 (2018).

[107] S. C. Kuhn and M. Richter, Combined Tensor Network/ Cluster Expansion Method Using Logic Gates: Illustrated for (Bi)Excitons by a Single-layer $\mathrm{MoS}_{2}$ Model System, Phys. Rev. B 99, 241301(R) (2019).

[108] S. C. Kuhn and M. Richter, Tensor Network Strategies for Calculating Biexcitons and Trions in Monolayer TwoDimensional Materials beyond the Ground State, Phys. Rev. B 101, 075302 (2020).

[109] A. V. Stier, N. P. Wilson, G. Clark, X. Xu, and S. A. Crooker, Probing the Influence of Dielectric Environment on Excitons in Monolayer $\mathrm{WSe}_{2}$ : Insight from High Magnetic Fields, Nano Lett. 16, 7054 (2016).

[110] I. Kylänpää and H.-P. Komsa, Binding Energies of Exciton Complexes in Transition Metal Dichalcogenide Monolayers and Effect of Dielectric Environment, Phys. Rev. B 92, 205418 (2015).

[111] C.-K. Yong, J. Horng, Y. Shen, H. Cai, A. Wang, C.-S. Yang, C.-K. Lin, S. Zhao, K. Watanabe, T. Taniguchi, S. Tongay, and F. Wang, Biexcitonic Optical Stark Effects in Monolayer Molybdenum Diselenide, Nat. Phys. 14, 1092 (2018).

[112] G. Plechinger, P. Nagler, J. Kraus, N. Paradiso, C. Strunk, C. Schüller, and T. Korn, Identification of Excitons, Trions and Biexcitons in Single-Layer $\mathrm{WS}_{2}$, Phys. Status Solidi (RRL) 9, 457 (2015).

[113] J. Shang, X. Shen, C. Cong, N. Peimyoo, B. Cao, M. Eginligil, and T. Yu, Observation of Excitonic Fine Structure in a 2D Transition-Metal Dichalcogenide Semiconductor, ACS Nano 9, 647 (2015).

[114] Y. You, X.-X. Zhang, T. C. Berkelbach, M. S. Hybertsen, D. R. Reichman, and T. F. Heinz, Observation of Biexcitons in Monolayer $\mathrm{WSe}_{2}$, Nat. Phys. 11, 477 (2015).

[115] M. S. Kim, S. J. Yun, Y. Lee, C. Seo, G. H. Han, K. K. Kim, Y. H. Lee, and J. Kim, Biexciton Emission from Edges and Grain Boundaries of Triangular $\mathrm{WS}_{2}$ Monolayers, ACS Nano 10, 2399 (2016).

[116] H. S. Lee, M. S. Kim, H. Kim, and Y. H. Lee, Identifying Multiexcitons in $\mathrm{MoS}_{2}$ Monolayers at Room Temperature, Phys. Rev. B 93, 140409(R) (2016).

[117] J. Pandey and A. Soni, Unraveling Biexciton and Excitonic Excited States from Defect Bound States in Monolayer $\mathrm{MoS}_{2}$, Appl. Surf. Sci. 463, 52 (2019).

[118] A. Knorr, T. Östreich, K. Schönhammer, R. Binder, and S. W. Koch, Asymptotic Analytic Solution for Rabi Oscillations in a System of Weakly Excited Excitons, Phys. Rev. B 49, 14024 (1994).

[119] R. Binder, S. W. Koch, M. Lindberg, N. Peyghambarian, and W. Schäfer, Ultrafast Adiabatic Following in Semiconductors, Phys. Rev. Lett. 65, 899 (1990).

[120] T. Östreich and A. Knorr, Various Appearances of Rabi Oscillations for $2 \pi$-Pulse Excitation in a Semiconductor, Phys. Rev. B 48, 17811 (1993).

[121] R. Binder and S. W. Koch, Nonequilibrium Semiconductor Dynamics, Prog. Quantum Electron. 19, 307 (1995).

[122] E. J. Mayer, G. O. Smith, V. Heuckeroth, J. Kuhl, K. Bott, A. Schulze, T. Meier, D. Bennhardt, S. W. Koch, 
P. Thomas, R. Hey, and K. Ploog, Evidence of Biexcitonic Contributions to Four-Wave Mixing in GaAs Quantum Wells, Phys. Rev. B 50, 14730 (1994).

[123] T. Voss, I. Rückmann, J. Gutowski, V. M. Axt, and T. Kuhn, Coherent Control of the Exciton and ExcitonBiexciton Transitions in the Generation of Nonlinear Wave-Mixing Signals in a Semiconductor Quantum Well, Phys. Rev. B 73, 115311 (2006).

[124] T. Voss, I. Rückmann, J. Gutowski, V. M. Axt, and T. Kuhn, Coherent Control of Exciton-Biexciton Beats: Direction Selectivity of Four-Wave-Mixing Signals in Experiment and Microscopic Theory, Phys. Status Solidi (b) 243, 2410 (2006).

[125] P. Borri, W. Langbein, S. Schneider, U. Woggon, R. L. Sellin, D. Ouyang, and D. Bimberg, Rabi Oscillations in the Excitonic Ground-State Transition of InGaAs Quantum Dots, Phys. Rev. B 66, 081306(R) (2002).

[126] J. M. Villas-Bôas, S. E. Ulloa, and A. O. Govorov, Decoherence of Rabi Oscillations in a Single Quantum Dot, Phys. Rev. Lett. 94, 057404 (2005).

[127] S. Stufler, P. Machnikowski, P. Ester, M. Bichler, V. M. Axt, T. Kuhn, and A. Zrenner, Two-Photon Rabi Oscillations in a Single $\mathrm{In}_{x} \mathrm{Ga}_{1-x}$ As/GaAs Quantum Dot, Phys. Rev. B 73, 125304 (2006).

[128] M. Glässl, A. M. Barth, and V. M. Axt, Proposed Robust and High-Fidelity Preparation of Excitons and Biexcitons in Semiconductor Quantum Dots Making Active Use of Phonons, Phys. Rev. Lett. 110, 147401 (2013).

[129] T. Unold, K. Mueller, C. Lienau, T. Elsaesser, and A. D. Wieck, Optical Control of Excitons in a Pair of Quantum Dots Coupled by the Dipole-Dipole Interaction, Phys. Rev. Lett. 94, 137404 (2005).

[130] J. Förstner, C. Weber, J. Danckwerts, and A. Knorr, PhononAssisted Damping of Rabi Oscillations in Semiconductor Quantum Dots, Phys. Rev. Lett. 91, 127401 (2003).

[131] J. Förstner, C. Weber, J. Danckwerts, and A. Knorr, Phonon-Induced Damping of Rabi Oscillations in Semiconductor Quantum Dots, Phys. Status Solidi (b) 238, 419 (2003).

[132] D. E. Reiter, S. Lüker, K. Gawarecki, A. Grodecka-Grad, P. Machnikowski, V. Axt, and T. Kuhn, Phonon Effects on Population Inversion in Quantum Dots: Resonant, Detuned and Frequency-Swept Excitations, Acta Phys. Pol. A 122, 1065 (2012).

[133] D. E. Reiter, T. Kuhn, M. Glässl, and V. M. Axt, The Role of Phonons for Exciton and Biexciton Generation in an Optically Driven Quantum Dot, J. Phys. Condens. Matter 26, 423203 (2014).

[134] Q. Q. Wang, A. Muller, P. Bianucci, E. Rossi, Q. K. Xue, T. Takagahara, C. Piermarocchi, A. H. MacDonald, and C. K. Shih, Decoherence Processes during Optical Manipulation of Excitonic Qubits in Semiconductor Quantum Dots, Phys. Rev. B 72, 035306 (2005).

[135] A. A. Mitioglu, K. Galkowski, A. Surrente, L. Klopotowski, D. Dumcenco, A. Kis, D. K. Maude, and P. Plochocka, Magnetoexcitons in Large Area CVD-Grown Monolayer $\mathrm{MoS}_{2}$ and $\mathrm{MoSe}_{2}$ on Sapphire, Phys. Rev. B 93, 165412 (2016).

[136] Y. Li, J. Ludwig, T. Low, A. Chernikov, X. Cui, G. Arefe, Y. D. Kim, A. M. van der Zande, A. Rigosi, H. M. Hill,
S. H. Kim, J. Hone, Z. Li, D. Smirnov, and T. F. Heinz, Valley Splitting and Polarization by the Zeeman Effect in Monolayer $\mathrm{MoSe}_{2}$, Phys. Rev. Lett. 113, 266804 (2014).

[137] G. Aivazian, Z. Gong, A. M. Jones, R.-L. Chu, J. Yan, D. G. Mandrus, C. Zhang, D. Cobden, W. Yao, and X. Xu, Magnetic Control of Valley Pseudospin in Monolayer $\mathrm{WSe}_{2}$, Nat. Phys. 11, 148 (2015).

[138] A. Srivastava, M. Sidler, A. V. Allain, D. S. Lembke, A. Kis, and A. Imamoğlu, Valley Zeeman Effect in Elementary Optical Excitations of Monolayer $\mathrm{WSe}_{2}$, Nat. Phys. 11, 141 (2015).

[139] D. MacNeill, C. Heikes, K. F. Mak, Z. Anderson, A. Kormányos, V. Zólyomi, J. Park, and D. C. Ralph, Breaking of Valley Degeneracy by Magnetic Field in Monolayer $\mathrm{MoSe}_{2}$, Phys. Rev. Lett. 114, 037401 (2015).

[140] G. Wang, L. Bouet, M. M. Glazov, T. Amand, E. L. Ivchenko, E. Palleau, X. Marie, and B. Urbaszek, Magneto-Optics in Transition Metal Diselenide Monolayers, 2D Mater. 2, 034002 (2015).

[141] G. Plechinger, P. Nagler, A. Arora, A. G. del Águila, M. V. Ballottin, T. Frank, P. Steinleitner, M. Gmitra, J. Fabian, P. C. M. Christianen, R. Bratschitsch, C. Schüller, and T. Korn, Excitonic Valley Effects in Monolayer $\mathrm{WS}_{2}$ under High Magnetic Fields, Nano Lett. 16, 7899 (2016).

[142] A. Arora, R. Schmidt, R. Schneider, M. R. Molas, I. Breslavetz, M. Potemski, and R. Bratschitsch, Valley Zeeman Splitting and Valley Polarization of Neutral and Charged Excitons in Monolayer $\mathrm{MoTe}_{2}$ at High Magnetic Fields, Nano Lett. 16, 3624 (2016).

[143] R. Schmidt, A. Arora, G. Plechinger, P. Nagler, A. G. del Águila, M. V. Ballottin, P. C. M. Christianen, S. M. de Vasconcellos, C. Schüller, T. Korn, and R. Bratschitsch, Magnetic-Field-Induced Rotation of Polarized Light Emission from Monolayer $\mathrm{WS}_{2}$, Phys. Rev. Lett. 117, 077402 (2016).

[144] A. V. Stier, K. M. McCreary, B. T. Jonker, J. Kono, and S. A. Crooker, Exciton Diamagnetic Shifts and Valley Zeeman Effects in Monolayer $\mathrm{WS}_{2}$ and $\mathrm{MoS}_{2}$ to 65 Tesla, Nat. Commun. 7, 10643 (2016).

[145] J. Zipfel, J. Holler, A. A. Mitioglu, M. V. Ballottin, P. Nagler, A. V. Stier, T. Taniguchi, K. Watanabe, S. A. Crooker, P. C. M. Christianen, T. Korn, and A. Chernikov, Spatial Extent of the Excited Exciton States in $\mathrm{WS}_{2}$ Monolayers from Diamagnetic Shifts, Phys. Rev. B 98, 075438 (2018).

[146] A. Arora, M. Koperski, A. Slobodeniuk, K. Nogajewski, R. Schmidt, R. Schneider, M. R. Molas, S. M. de Vasconcellos, R. Bratschitsch, and M. Potemski, Zeeman Spectroscopy of Excitons and Hybridization of Electronic States in Few-Layer WSe ${ }_{2}, \mathrm{MoSe}_{2}$ and $\mathrm{MoTe}_{2}, 2 \mathrm{D}$ Mater. 6, 015010 (2018).

[147] J. Bechhoefer, Feedback for Physicists: A Tutorial Essay on Control, Rev. Mod. Phys. 77, 783 (2005).

[148] U. Dorner and P. Zoller, Laser-Driven Atoms in HalfCavities, Phys. Rev. A 66, 023816 (2002).

[149] A. Carmele, J. Kabuss, F. Schulze, S. Reitzenstein, and A. Knorr, Single Photon Delayed Feedback: A Way to Stabilize Intrinsic Quantum Cavity Electrodynamics, Phys. Rev. Lett. 110, 013601 (2013). 
[150] S. M. Hein, F. Schulze, A. Carmele, and A. Knorr, Optical Feedback-Enhanced Photon Entanglement from a Biexciton Cascade, Phys. Rev. Lett. 113, 027401 (2014).

[151] K. Barkemeyer, R. Finsterhölzl, A. Knorr, and A. Carmele, Revisiting Quantum Feedback Control: Disentangling the Feedback-Induced Phase from the Corresponding Amplitude, Adv. Quantum Technol. 3, 1900078 (2020).

[152] G. Scuri, Y. Zhou, A. A. High, D. S. Wild, C. Shu, K. De Greve, L. A. Jauregui, T. Taniguchi, K. Watanabe, P. Kim, M. D. Lukin, and H. Park, Large Excitonic Reflectivity of Monolayer $\mathrm{MoSe}_{2}$ Encapsulated in Hexagonal Boron Nitride, Phys. Rev. Lett. 120, 037402 (2018).

[153] H. H. Fang, B. Han, C. Robert, M. A. Semina, D. Lagarde, E. Courtade, T. Taniguchi, K. Watanabe, T. Amand, B. Urbaszek, M. M. Glazov, and X. Marie, Control of the Exciton Radiative Lifetime in van der Waals Heterostructures, Phys. Rev. Lett. 123, 067401 (2019).

[154] J. Horng, Y.-H. Chou, T.-C. Chang, C.-Y. Hsu, T.-C. Lu, and H. Deng, Engineering Radiative Coupling of Excitons in 2D Semiconductors, Optica 6, 1443 (2019).

[155] Y. Zhou, G. Scuri, J. Sung, R. J. Gelly, D. S. Wild, K. De Greve, A. Y. Joe, T. Taniguchi, K. Watanabe, P. Kim, M. D. Lukin, and H. Park, Controlling Excitons in an Atomically Thin Membrane with a Mirror, Phys. Rev. Lett. 124, 027401 (2020).

[156] C. Rogers, D. Gray, Jr., N. Bogdanowicz, T. Taniguchi, K. Watanabe, and H. Mabuchi, Coherent Feedback Control of Two-Dimensional Excitons, Phys. Rev. Research 2, 012029 (2020).

[157] I. Epstein, B. Terres, A. J. Chaves, V.-V. Pusapati, D. A. Rhodes, B. Frank, V. Zimmermann, Y. Qin, K. Watanabe, T. Taniguchi, H. Giessen, S. Tongay, J. C. Hone, N. M. R. Peres, and F. H. L. Koppens, Near-Unity Light Absorption in a Monolayer $\mathrm{WS}_{2}$ van der Waals Heterostructure Cavity, Nano Lett. 20, 3545 (2020).

[158] J. Horng, E. W. Martin, Y.-H. Chou, E. Courtade, T.-C. Chang, C.-Y. Hsu, M.-H. Wentzel, H. G. Ruth, T.-C. Lu, S. T. Cundiff, F. Wang, and H. Deng, Perfect Absorption by an Atomically Thin Crystal, Phys. Rev. Applied 14, 024009 (2020).

[159] C. Schneider, M. M. Glazov, T. Korn, S. Höfling, and B. Urbaszek, Two-Dimensional Semiconductors in the Regime of Strong Light-Matter Coupling, Nat. Commun. 9, 2695 (2018).

[160] M. Combescot and O. Betbeder-Matibet, Biexciton Oscillator Strength, Phys. Rev. B 80, 205313 (2009).

[161] R. Trebino, K. W. DeLong, D. N. Fittinghoff, J. N. Sweetser, M. A. Krumbügel, B. A. Richman, and D. J. Kane, Measuring Ultrashort Laser Pulses in the TimeFrequency Domain Using Frequency-Resolved Optical Gating, Rev. Sci. Instrum. 68, 3277 (1997).
[162] F. A. Rasmussen and K. S. Thygesen, Computational 2D Materials Database: Electronic Structure of TransitionMetal Dichalcogenides and Oxides, J. Phys. Chem. C 119, 13169 (2015).

[163] A. Kormányos, G. Burkard, M. Gmitra, J. Fabian, V. Zólyomi, N. D. Drummond, and V. Fal'ko, $k \cdot p$ Theory for Two-Dimensional Transition Metal Dichalcogenide Semiconductors, 2D Mater. 2, 022001 (2015).

[164] A. Kumar and P. K. Ahluwalia, Tunable Dielectric Response of Transition Metals Dichalcogenides $\mathrm{MX}_{2}$ $(\mathrm{M}=\mathrm{Mo}, W ; \mathrm{X}=\mathrm{S}, \mathrm{Se}, \mathrm{Te})$ : Effect of Quantum Confinement, Physica (Amsterdam) 407B, 4627 (2012).

[165] The phonon-mediated dephasing $\gamma_{0}$ is calculated according to the methods given in Ref. [56].

[166] S. Brem, J. Zipfel, M. Selig, A. Raja, L. Waldecker, J. Ziegler, T. Taniguchi, K. Watanabe, A. Chernikov, and E. Malić, Intrinsic Lifetime of Higher Excitonic States in Tungsten Diselenide Monolayers, Nanoscale 11, 12381 (2019).

[167] C. Robert, H. Dery, L. Ren, D. van Tuan, E. Courtade, M. Yang, B. Urbaszek, D. Lagarde, K. Watanabe, T. Taniguchi, T. Amand, and X. Marie, Measurement of Conduction and Valence Bands g-Factors in a Transition Metal Dichalcogenide Monolayer, arXiv:2008.07464.

[168] A. Kormányos, P. Rakyta, and G. Burkard, Landau Levels and Shubnikov-de Haas Oscillations in Monolayer Transition Metal Dichalcogenide Semiconductors, New J. Phys. 17, 103006 (2015).

[169] D. V. Rybkovskiy, I. C. Gerber, and M. V. Durnev, Atomically Inspired $k \cdot p$ Approach and Valley Zeeman Effect in Transition Metal Dichalcogenide Monolayers, Phys. Rev. B 95, 155406 (2017).

[170] T. Woźniak, P. E. F. Junior, G. Seifert, A. Chaves, and J. Kunstmann, Exciton g Factors of van der Waals Heterostructures from First Principles Calculations, Phys. Rev. B 101, 235408 (2020).

[171] T. Deilmann, P. Krüger, and M. Rohlfing, Ab Initio Studies of Exciton g Factors: Monolayer Transition Metal Dichalcogenides in Magnetic Fields, Phys. Rev. Lett. 124, 226402 (2020).

[172] J. Förste, N. V. Tepliakov, S. Y. Kruchinin, J. Lindlau, V. Funk, M. Förg, K. Watanabe, T. Taniguchi, A. S. Baimuratov, and A. Högele, Exciton g-Factors in Monolayer and Bilayer $\mathrm{WSe}_{2}$ from Experiment and Theory, Nat. Commun. 11, 4539 (2020).

[173] F. Katsch, D. Christiansen, R. Schmidt, S. M. de Vasconcellos, R. Bratschitsch, A. Knorr, and M. Selig, Theory of the Coherent Response of Magneto-Excitons and Magneto-Biexcitons in Monolayer Transition Metal Dichalcogenides, Phys. Rev. B 102, 115420 (2020). 